\title{
Investigating Nonlinear Dependence between Climate Fields $\mathscr{O}$
}

\author{
MATT J. FISCHER \\ Australian Nuclear Science and Technology Organisation, Lucas Heights, New South Wales, Australia
}

(Manuscript received 29 July 2016, in final form 20 March 2017)

\begin{abstract}
The Earth's ice-ocean-atmosphere system exhibits nonlinear responses, such as the difference in the magnitude of the atmospheric response to positive or negative ocean or sea ice anomalies. Two classes of methods that have previously been used to investigate the nonlinear dependence between climate fields are kernel methods and neural network methods. In this paper, a third methodology is introduced: gradient-based kernel dimension reduction. Gradient-based kernel methods are an extension of conventional kernel methods, but gradient-based methods focus on the directional derivatives of the regression surface between two fields. Specifically, a new gradient-based method is developed here: gradient kernel canonical correlation analysis (gKCCA). In gKCCA, the canonical directions maximize the directional derivatives between the predictor field and the response field, while the canonical components of the response field maximize the correlation with a nonlinear augmentation of the predictor canonical components. Gradient-based kernel methods have several advantages: their components can be directly related to the original fields (unlike in conventional kernel methods), and the projection vectors are determined by analytical solution (unlike in neural networks). Here gKCCA is applied to the question of nonlinear coupling between high-frequency (2-3 years) and low-frequency (4-6 years) modes in the Pacific Ocean. The leading gKCCA subspace shows a significant nonlinear coupling between the low-pass and high-pass fields. The paper also shows that the results of gKCCA are robust to different levels of noise and different kernel functions.
\end{abstract}

\section{Introduction}

The identification of coupled patterns is important to many areas of climate research, including teleconnection and attribution studies (Smoliak et al. 2015), seasonal climate forecasting (Wilks 2008), climate field reconstruction using proxy records (Smerdon et al. 2011), and linear inverse modeling (DelSole and Tippett 2008). While early research focused on the linear dependence between two climate fields (Bretherton et al. 1992; Tippett et al. 2008), other studies have also recognized the importance of nonlinear dependence (Ramírez et al. 2006; Cannon and Hsieh 2008; Ortiz Beviá et al. 2010; Evans et al. 2014).

Two main methods have previously been used to investigate the nonlinear coupling between two fields: neural net canonical correlation analysis (NNCCA)

Supplemental information related to this paper is available at the Journals Online website: http://dx.doi.org/10.1175/ JCLI-D-16-0563.s1.

Corresponding author: Matt J. Fischer, mjf@ansto.gov.au
(Hsieh 2001; Wu et al. 2005; Cannon and Hsieh 2008) and kernel canonical correlation analysis (KCCA) (Arenas-García et al. 2013). In NNCCA, parametric mapping functions are used to link one-dimensional projections of the climate fields, while in KCCA, the climate fields are abstractly mapped into a higherdimensional space (or feature space) and linear canonical correlation analysis (CCA) is performed in the feature space. KCCA has not been widely used in the climate sciences (perhaps for the reason below), but Lima et al. (2009) used the related method of kernel principal component analysis (KPCA). Both NNCCA and KCCA have issues. For KCCA (and KPCA), the time components are not linear combinations of the predictor field or the response field, which can make the KCCA (KPCA) components difficult to interpret. For NNCCA, the projection weights are found by optimization methods, and NNCCA typically relies on iterative decomposition (i.e., the components are extracted one at a time), so NNCCA is computationally expensive. Also, NNCCA is not appropriate for high-dimensional data and hence is typically applied to a low-dimensional principal component subspace of the original data fields. 
In both methods, the kernel (or mapping) functions are generally arbitrarily chosen.

The main aims of this study are to develop a new method for investigating nonlinear dependence between two climate fields and to illustrate the method by examples. The new method, gradient-based kernel canonical correlation analysis (gKCCA), builds on the ideas of Fukumizu and Leng (2014), who introduced gradient-based kernel regression with a univariate response variable. The following paper describes a gradient-based kernel method for coupled fields (section 2) and also its connections with CCA (sections 2a, $2 \mathrm{~b}$, and 2d) and the linear model (section 2c). Cross validation for nonlinear models is discussed in section 3. Studies of the tropical Pacific Ocean have suggested that the system oscillates at preferred frequencies, such as annual, quasi-biennial (QB), and quasiquadrennial (QQ) frequencies (Bejarano and Jin 2008), but the nature, and the spatial structure, of the coupling between these modes remains unclear. In section 4 , the gKCCA method is used to investigate nonlinear coupling between modes with different frequencies in the Pacific Ocean. Finally, the sensitivity of the gKCCA method with respect to different levels of noise and different kernel functions is investigated (section 5).

\section{Methods}

\section{a. Overview}

Given two different space-time anomaly fields $\mathbf{X}$ and $\mathbf{Y}$, it is often useful to ask whether there are a small number of coupled patterns that link the two fields. Canonical correlation analysis is a popular method for investigating linear dependence between two fields. CCA is based on the following model:

$$
\begin{aligned}
& \mathbf{Y A}=\mathbf{X W D} \text { and } \\
& \hat{\mathbf{Y}}=\mathbf{Y} \mathbf{A}\left(\mathbf{P}^{Y}\right)^{\prime}, \quad \hat{\mathbf{X}}=\mathbf{X W}\left(\mathbf{P}^{X}\right)^{\prime}, \\
& \text { subject to } \mathbf{W}^{\prime} \mathbf{\Sigma}_{X} \mathbf{W}=\mathbf{I}_{d} \text { and } \quad \mathbf{A}^{\prime} \mathbf{\Sigma}_{Y} \mathbf{A}=\mathbf{I}_{d},
\end{aligned}
$$

where $\mathbf{P}_{j}^{X}$ and $\mathbf{P}_{j}^{Y}$ are a pair of canonical spatial patterns, $\mathbf{Y A}_{j}$ and $\mathbf{X} \mathbf{W}_{j}$ are a pair of canonical time series, the single subscript $j$ in $\mathbf{P}_{j}^{X}$, for example, denotes the $j$ th column vector of matrix $\mathbf{P}^{X}$, except in the case of $\mathbf{I}_{d}$, which is an identity matrix of rank $d$. The prime symbol denotes matrix transposition, and $\hat{\mathbf{X}}$ and $\hat{\mathbf{Y}}$ are the predicted (reconstructed) fields. The term $\mathbf{X}$ is a $n \times p$ anomaly field, $\mathbf{W}$ is a $p \times d$ matrix of projection vectors, $\mathbf{Y}$ is an $n \times q$ anomaly field, $\mathbf{A}$ is a $q \times d$ matrix of projection vectors, and $\mathbf{P}$ is a ( $p$ or $q) \times d$ matrix, where $d \leq \min (p, q)$ (see Table 1 for notation hereinafter).
TABLE 1. Notation used.

\begin{tabular}{ll}
\hline \hline Symbol & \multicolumn{1}{c}{ Definition } \\
\hline $\mathbf{A}$ & $q \times d$ matrix of weights (or loadings) \\
$\mathbf{D}$ & $d \times d$ diagonal matrix of singular values \\
$\mathbf{E}$ & $n \times d$ matrix of residual values \\
$\Phi(\cdot)$ & A mapping function \\
$\phi(\cdot)$ & $n \times$ df basis matrix \\
$\mathbf{G}$ & $n \times n$ Gram matrix \\
$\mathbf{H}$ & $n \times q$ matrix of linear coefficients \\
$\mathbf{I}_{p}$ & Identity matrix of rank $p$ \\
$K(\cdot, \cdot)$ & Kernel function \\
$\mathbf{L}$ & Diagonal matrix of eigenvalues; $\mathbf{L}=\mathbf{\mathbf { D } ^ { 2 }}$ \\
$\mathbf{P}$ & $(p$ or $q) \times d$ matrix of spatial patterns \\
$\mathbf{R}$ & $n \times d$ matrix of canonical time series; $\mathbf{R}=\mathbf{X W}$ \\
$\mathbf{\Sigma}_{X}$ & Covariance matrix of the subscripted variables \\
$\operatorname{tr}[\cdot]$ & Matrix trace, e.g., tr[X'X]= $\mathbf{X} \|^{2}$ \\
$\mathbf{T}$ & $n \times d$ matrix of canonical time series; $\mathbf{T}=\mathbf{Y A}$ \\
$\mathbf{V}$ & $(p$ or $q) \times d$ matrix of eigenvectors \\
$\mathbf{W}$ & $p \times d$ matrix of weights $($ or loadings) \\
$\mathbf{X}$ & $n \times p$ field; $p$ stations with $n$ time points \\
$\mathbf{Y}$ & $n \times q$ field \\
\hline
\end{tabular}

The canonical correlations $\rho$ are the diagonal elements of the $d \times d$ matrix $\mathbf{D}=\mathbf{W}^{\prime} \mathbf{\Sigma}_{X Y} \mathbf{A}$.

CCA is a useful method for two reasons. The first is that the canonical time components are marginal subspaces, or one-dimensional projections of the fields. The advantage of this is that CCA models a multidimensional regression surface by the simple addition of some marginal subspaces; for example,

$$
\hat{\mathbf{Y}}_{k}=\rho_{1} \mathbf{P}_{k 1}^{Y} \mathbf{X} \mathbf{W}_{1}+\cdots+\rho_{d} \mathbf{P}_{k d}^{Y} \mathbf{X} \mathbf{W}_{d},
$$

where the joint subscript $k d$, for example, denotes the element at row $k$ and column $d$ in matrix $\mathbf{P}^{Y}$. Each component $\mathbf{X} \mathbf{W}_{j}$ forms one edge of a hyperplane, and the hyperplane is modeled as the sum of linear functions that make up its edges (hence the term marginal functions or marginal subspaces). Modeling a high-dimensional function is difficult because of the "curse of dimensionality"; that is, points are always sparse in high dimensions (e.g., Scott 2015), but it is less difficult to model a high-dimensional function by using marginal functions because marginal functions are low-dimensional projections of the high-dimensional function. The second reason that CCA is useful is that CCA seeks component pairs that have a high correlation; that is, CCA seeks to find projection matrices $\mathbf{W}$ and $\mathbf{A}$ that maximize a particular criterion:

$$
\iota_{\mathrm{CCA}}=\operatorname{cor}\left(\mathbf{X W}_{j}, \mathbf{Y} \mathbf{A}_{j}\right)^{2},
$$

where $\iota_{\mathrm{CCA}}$ is the criterion to be maximized. This focus on correlation means that CCA can find coupled 
patterns that do not explain a large proportion of the variability in the predictor or response fields, and hence CCA can find signals in noisy fields.

The main disadvantage with CCA is its focus on linear correlation, which means it may miss structures that have a nonlinear dependence. Thus it would be nice to have a method in which the advantages of CCA were adopted to the nonlinear setting. Such a method might aim to model a nonlinear regression surface as follows:

$$
\mathbf{Y A}=\left[\begin{array}{lll}
f_{1}\left(\mathbf{X W}_{1}\right) & \ldots & f_{d}\left(\mathbf{X} \mathbf{W}_{d}\right)
\end{array}\right],
$$

subject to $\mathbf{W} \boldsymbol{\Sigma}_{X} \mathbf{W}=\mathbf{I}_{d}$ and $\mathbf{A}^{\prime} \boldsymbol{\Sigma}_{Y} \mathbf{A}=\mathbf{I}_{d}$. It also might aim to maximize the following criterion of interest:

$$
\iota_{\mathrm{nICCA}}=\operatorname{cor}\left[f_{j}\left(\mathbf{X} \mathbf{W}_{j}\right), \mathbf{Y} \mathbf{A}_{j}\right]^{2} .
$$

Equation (4) is a nonlinear extension of Eq. (1a): the functions $f_{j}\left(\mathbf{X W}_{j}\right)$ form the edges of a manifold (a curved sheet). The main advantage of this particular nonlinear extension, as with linear CCA, is to model the sheet as the sum of functions that describe the edges of the sheet:

$$
\hat{\mathbf{Y}}=g(\mathbf{X})=f_{1}\left(\mathbf{X} \mathbf{W}_{1}\right)\left(\mathbf{P}_{1}^{Y}\right)^{\prime}+\cdots+f_{d}\left(\mathbf{X} \mathbf{W}_{d}\right)\left(\mathbf{P}_{d}^{Y}\right)^{\prime}
$$

This model covers a subset of all possible nonlinear functions, but further extensions are possible, for example, by including interaction terms such as $f_{j}\left(\mathbf{X} \mathbf{W}_{j}\right) f_{k}\left(\mathbf{X} \mathbf{W}_{k}\right)$ (which will be reported elsewhere). Hereinafter, it is assumed that $g(\mathbf{X})$ is a locally differentiable function (i.e., gradient vectors can be estimated at points) and that one point in $\mathbf{X}$-space maps onto one point in $\mathbf{Y}$-space.

The gKCCA method has two steps:

1) Find $\mathbf{X W}$, the subspace of $\mathbf{X}$ that is linearly or nonlinearly related to $\mathbf{Y}$.

2) Model the functions $f_{j}\left(\mathbf{X} \mathbf{W}_{j}\right)$ and find $\mathbf{Y A}$, by performing CCA on $\mathbf{Y}$ and a nonlinear augmentation of $\mathbf{X W}$.

Step 1 and step 2 are explained in sections $2 \mathrm{~b}$ and $2 \mathrm{~d}$, respectively.

\section{$b$. The gradient field and its eigenvectors (or estimating $\mathbf{W}$}

This section explains how to do step 1 and illustrates it using a simulated example. Some extra details of the method are found in appendix A and supplementary sections 1-3, and cross-references are given as necessary.
In the example here, a nonlinear sheet is simulated with a flat edge $\mathbf{X} \mathbf{W}_{2}$ and a curvy edge $f\left(\mathbf{X} \mathbf{W}_{1}\right)$, and an error $\mathbf{E}$ is added to the sheet:

$$
\begin{aligned}
\mathbf{Y} & =6.3 f\left(\mathbf{X} \mathbf{W}_{1}\right)+0\left(\mathbf{X} \mathbf{W}_{2}\right)+\mathbf{E}, \quad \text { and } \\
f\left(\mathbf{X} \mathbf{W}_{1}\right) & =\exp \left(-0.1 \mathbf{X} \mathbf{W}_{1}\right)\left(\mathbf{X} \mathbf{W}_{1}\right)^{2},
\end{aligned}
$$

where $\mathbf{Y}$ is a $n \times 1$ vector, $\mathbf{x}_{i} \sim \mathscr{Q} 6[\min =(-15-15)$, $\max =(1515)]$, where $\mathscr{C}$ is a multivariate uniform random distribution spanning the given range, and $\mathbf{x}_{i}$ is a row vector of $\mathbf{X}, i=(1, \ldots, n)$. So in this example, $\mathbf{X}$ is a $n \times 2$ matrix, $\mathbf{W}$ is a $2 \times 2$ matrix, and $\mathbf{E}$ is an $n \times 1$ vector. The terms $\mathbf{X}$ and $\mathbf{Y}$ were also centered and scaled to unit variance. Thus $\mathbf{X}_{1}, \mathbf{X}_{2}$ and $\mathbf{Y}$ form a curved sheet in a three-dimensional space (Fig. 1a). Of course, it is possible to use linear CCA to try to model this surface as a linear sheet (a plane). But if the fitted CCA model was used to predict $\mathbf{Y}$, then it would be apparent that the linear sheet model fails to properly predict $\mathbf{Y}$.

So, given only $\mathbf{X}$ and $\mathbf{Y}$, the aim here is to find the directional vector or vectors $\mathbf{W}$ that capture the nonlinearity in the curved sheet, as a set of one-dimensional projections. In the example in Eq. (7), all the nonlinearity can be captured in one direction $\mathbf{W}_{1}$. The first question to be answered is how to find those directions.

One solution is to find the directions that maximize the gradient of $f_{j}\left(\mathbf{X} \mathbf{W}_{j}\right)$ given $\mathbf{Y}=g(\mathbf{X})=\boldsymbol{\Sigma} f_{j}\left(\mathbf{X} \mathbf{W}_{j}\right)\left(\mathbf{P}_{j}^{Y}\right)^{\prime}$. Supplementary section 1 contains further information about gradients and directional derivatives, but a less technical narrative follows. Assuming that gradients can be calculated at various points of a function, there should be directions that maximize the mean (absolute or squared) gradient of the projected points along that direction. For any linear or nonlinear regression surface, the directions in which $\mathbf{X}$ has the greatest influence on $\mathbf{Y}$ must be associated with the steepest absolute gradients, while the directions in which $\mathbf{X}$ has the least influence on $\mathbf{Y}$ must be associated with the least absolute gradients. The advantage of working with gradients is that the solution can be cast as an eigenvalue problem with an analytical solution (supplementary section 1 ). No neural networks are necessary, and the analytical solution is computationally efficient.

To find the directions in which the average absolute gradient is the steepest, an estimate of the gradient field $\nabla g(\cdot)$ is required. The gradient field is made up of the gradient vectors at each point, where the gradient vector is $\nabla g(\mathbf{x})^{\prime}=\left[\partial g(\mathbf{x}) / \partial\left(x_{1}\right), \ldots, \partial g(\mathbf{x}) / \partial\left(x_{p}\right)\right]$ and $\mathbf{x}=$ $\left[x_{1}, \ldots, x_{p}\right]$. Estimating $\nabla g$ seems to require an estimate of $g(\mathbf{X})$, which is not known a priori. However there is an elegant way to estimate $\nabla g$, without explicitly calculating $g(\mathbf{X})$, by making use of kernel feature spaces. 
a)
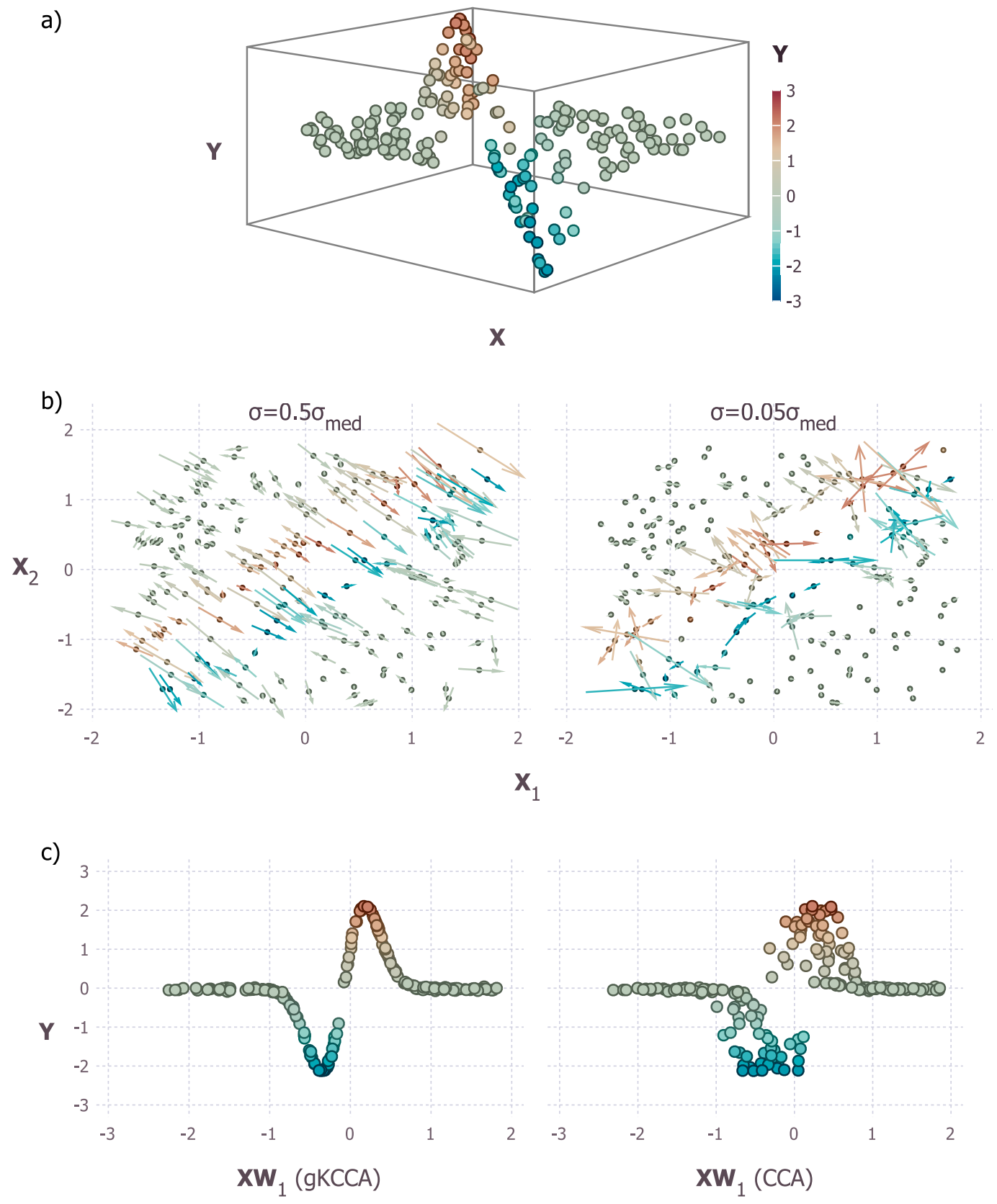

FIG. 1. The gKCCA method applied to a simulated example. (a) The simulated manifold [Eq. (7)]. (b) The gradient field [Eq. (8)] estimated using two different values of $\sigma$. (c) The leading component for (left) gKCCA and (right) CCA. In all plots, the color corresponds to the value of $\mathbf{Y}$ at that particular point.

A feature space is a higher-dimensional space into which points $\mathbf{x}_{i}$ are mapped, with mapping functions $\Phi\left(\mathbf{x}_{i}\right)$. Supplementary section 2 provides an example of a feature space. In the feature space, the number of points $n$ is the same as in the input space, but the number of variables $p$ can be much greater than in the input space. For some feature spaces, the inner product of the vector of mapping functions is a kernel function; that is, $K\left(\mathbf{x}_{i}, \mathbf{x}_{j}\right)=\Phi\left(\mathbf{x}_{i}\right) \Phi\left(\mathbf{x}_{j}\right)^{\prime}$ (see appendix A). If this is the case, then instead of writing $g\left(\mathbf{x}_{i}\right)=\Phi\left(\mathbf{x}_{i}\right) \mathbf{B}$, we can write $g\left(\mathbf{x}_{i}\right)=K\left(\mathbf{x}_{i}, \cdot\right) \mathbf{H}$, where $K\left(\mathbf{x}_{i}, \cdot\right)=\left[K\left(\mathbf{x}_{i}, \mathbf{x}_{1}\right), \ldots, K\left(\mathbf{x}_{i}, \mathbf{x}_{n}\right)\right]$, and $\mathbf{B}$ and $\mathbf{H}$ are matrices of linear coefficients (see supplementary section 3). That is, by using a kernel function, a nonlinear regression model can be calculated without explicitly calculating $\Phi(\mathbf{X})$. Similarly, the gradient vectors of the regression surface $g(\cdot)$ can be estimated 
by using the gradient vectors of the kernel function $K(\cdot, \cdot)$ :

$$
\nabla g\left(\mathbf{x}_{i}\right)=\nabla K\left(\mathbf{x}_{i}, \cdot\right) \mathbf{H},
$$

where $\nabla g\left(\mathbf{x}_{i}\right)$ is a $p \times q$ gradient matrix, $\nabla K\left(\mathbf{x}_{i}, \cdot\right)=$ $\left[\nabla K\left(\mathbf{x}_{i}, \mathbf{x}_{1}\right)^{\prime} \ldots \nabla K\left(\mathbf{x}_{i}, \mathbf{x}_{n}\right)^{\prime}\right]$ is a $p \times n$ matrix, and $\mathbf{H}$ is a $n \times q$ matrix of linear coefficients (as in supplementary section 3).

An example of a kernel function that will be used in this paper is the well-known Gaussian kernel:

$$
K_{g}\left(\mathbf{x}_{i}, \mathbf{x}_{j}\right)=\exp \left(-\frac{\left\|\mathbf{x}_{i}-\mathbf{x}_{j}\right\|^{2}}{2 \sigma^{2}}\right) .
$$

Its gradient vector is

$$
\nabla K_{g}\left(\mathbf{x}_{i}, \mathbf{x}_{j}\right)=\sigma^{-2} \exp \left(-\frac{\left\|\mathbf{x}_{i}-\mathbf{x}_{j}\right\|^{2}}{2 \sigma^{2}}\right)\left(\mathbf{x}_{i}-\mathbf{x}_{j}\right) .
$$

When the Gaussian kernel is used in Eq. (8), the gradient field at a particular point is modeled as a weighted sum of multivariate Gaussian kernels centered on the other points. The smoothness of the gradient field thus depends on the parameter $\sigma$ [Eqs. (9) and (10)]. It is convenient to reexpress this parameter in terms of the median Euclidean distance $\sigma_{\text {med }}$ between all the points in $\mathbf{X}$ :

$$
\begin{aligned}
\sigma & =c \sigma_{\text {med }}, \quad \text { and } \\
\sigma_{\text {med }} & =\underset{\text { all } i, j}{\operatorname{median}}\left[\ldots,\left\|\mathbf{x}_{i}-\mathbf{x}_{j}\right\|, \ldots\right],
\end{aligned}
$$

where $i \neq j$.

The gradient field for the example [Eq. (7)], calculated using Eq. (8) for two different values of $\sigma$, is shown in Fig. 1b. For $\sigma=0.5 \sigma_{\mathrm{med}}$, in the regions of steepest gradient, the gradient vectors are generally aligned along the same axial direction. For $\sigma=0.05 \sigma_{\text {med }}$, the gradient vectors are more scattered, because the Gaussian kernel is tighter and thus is more susceptible to random fluctuations in the field, caused by both $\mathbf{E}$ and the random spacing of points. In the other extreme (i.e., for large values of $c \sigma_{\text {med }}$ ), the modeled sheet becomes a hyperplane; that is, the modeled surface is the same as the linear CCA model.

Having estimated the gradient field [Eq. (8)], the estimation of $\mathbf{W}$ is straightforward. The directions in which the gradient field is steepest are simply the leading eigenvectors of the average of the outer product of the gradient vectors at each point:

$$
n^{-1} \sum_{i=1}^{n}\left[\nabla g\left(\mathbf{x}_{i}\right) \nabla g\left(\mathbf{x}_{i}\right)^{\prime}\right]=\mathbf{V L V}^{\prime} \text {. }
$$

Then $\mathbf{W}=\mathbf{V}$. Note that if $\mathbf{X}$ was whitened (i.e., $\mathbf{X} \boldsymbol{\Sigma}_{X}^{-0.5}$ ) prior to analysis, then $\mathbf{W}=\sum_{X}^{-0.5} \mathbf{V}$. The diagonal elements of $\mathbf{L}$ are equal to the mean-squared directional derivatives $\nabla g\left(\mathbf{x}_{i}\right)^{\prime} \mathbf{V}$ in the direction of each unit vector in $\mathbf{V}$.

Both linear CCA and gKCCA [Eqs. (8)-(12)] were used to estimate $\mathbf{W}$ for the example [Eq. (7)], and the leading direction for each method is shown in Fig. 1c. Using $\sigma=0.5 \sigma_{\text {med }}$, gKCCA accurately estimates the direction (Fig. 1b, left plot) and the nonlinear function (Fig. 1c, left) that forms an edge of the curved sheet (Fig. 1a). Linear CCA, though, finds the direction for which $\mathbf{X} \mathbf{W}_{1}$ and $\mathbf{Y}$ have the strongest linear correlation (Fig. 1c, right), but in this case the linear correlation is simply a correlation between two groups of points (low and high points). Further, the gKCCA model is more capable than the CCA model of predicting $\mathbf{Y}$ from $\mathbf{X}$, because for CCA the predicted values $\hat{\mathbf{Y}}$ will lie on a linear sheet, not a curved sheet.

\section{c. The linear model in kernel form}

This subsection shows how the linear model and its gradient vector can be expressed in kernel form, in order to provide an analytical example of the methods above. The well-known linear function $\mathbf{y}=L(\mathbf{x})$ can be expressed as follows:

$$
L\left(\mathbf{x}_{i}\right)=\mathbf{x}_{i}\left(\mathbf{X}^{\prime} \mathbf{X}+\lambda \mathbf{I}_{p}\right)^{-1} \mathbf{X}^{\prime} \mathbf{Y} .
$$

Its gradient vector (or gradient matrix, in the case that $q>1$ ) at all points can be expressed as follows:

$$
\nabla L\left(\mathbf{x}_{i}\right)=\left(\mathbf{X}^{\prime} \mathbf{X}+\lambda \mathbf{I}_{p}\right)^{-1} \mathbf{X}^{\prime} \mathbf{Y},
$$

where $\lambda$ is a ridge parameter as in ridge regression (see, e.g., Cannon 2009). The effect of the ridge parameter is that higher values of the parameter will give more weight to the principal components of the predictor matrix that have large eigenvalues, leading to less overfitting of the response.

Now the linear model and its gradient vector will be reexpressed in kernel form, using the linear kernel. The linear kernel is

$$
K_{1}\left(\mathbf{x}_{i}, \mathbf{x}_{j}\right)=\mathbf{x}_{i} \mathbf{x}_{j}^{\prime},
$$

and its gradient vector is

$$
\nabla K_{1}\left(\mathbf{x}_{i}, \mathbf{x}_{j}\right)=\mathbf{x}_{j} .
$$

Using the identity $\mathbf{X}^{\prime}\left(\mathbf{X} \mathbf{X}^{\prime}+\lambda \mathbf{I}_{n}\right)^{-1} \mathbf{Y}=\left(\mathbf{X}^{\prime} \mathbf{X}+\lambda \mathbf{I}_{p}\right)^{-1} \mathbf{X}^{\prime} \mathbf{Y}$ (see supplementary section 3 ), the linear model [Eq. (13)] can be reexpressed 


$$
L\left(\mathbf{x}_{i}\right)=K_{1}\left(\mathbf{x}_{i}, \cdot\right)\left(\mathbf{G}_{X}+n \varepsilon \mathbf{I}_{n}\right)^{-1} \mathbf{Y},
$$

where $K_{1}\left(\mathbf{x}_{i}, \cdot\right)=\left[\mathbf{x}_{i} \mathbf{x}_{1}^{\prime}, \ldots, \mathbf{x}_{i} \mathbf{x}_{n}^{\prime}\right]$.

The term $\mathbf{G}_{X}$ is a $n \times n$ Gram matrix for $\mathbf{X}$ where the elements of $\mathbf{G}_{X}$ are in the linear case $\mathbf{G}_{i j}=K_{\mathbf{l}}\left(\mathbf{x}_{i}, \mathbf{x}_{j}\right.$ ), and $\varepsilon$ is a ridge parameter (which has the same effect as $\lambda$ above). The gradient vector of the linear model [Eq. (14)] can now be reexpressed as follows:

$$
\begin{aligned}
\nabla L\left(\mathbf{x}_{i}\right) & =\nabla K_{1}\left(\mathbf{x}_{i}, \cdot\right)\left(\mathbf{G}_{X}+n \varepsilon \mathbf{I}_{n}\right)^{-1} \mathbf{Y} \\
& =\mathbf{X}^{\prime}\left(\mathbf{X X}^{\prime}+n \varepsilon \mathbf{I}_{n}\right)^{-1} \mathbf{Y},
\end{aligned}
$$

where $\nabla K_{1}\left(\mathbf{x}_{i}, \cdot\right)=\mathbf{X}^{\prime}$.

The advantage of Eqs. (17) and (18) is that these equations can be generalized to the nonlinear case (supplementary section 3 ), and the gradient vectors of many nonlinear $g(\cdot)$ can be calculated as a linear combination of the kernel gradient vectors:

$$
\begin{aligned}
g\left(\mathbf{x}_{i}\right) & =K\left(\mathbf{x}_{i}, \cdot\right)\left(\mathbf{G}_{X}+n \varepsilon \mathbf{I}_{n}\right)^{-1} \mathbf{Y}, \quad \text { and } \\
\nabla g\left(\mathbf{x}_{i}\right) & =\nabla K\left(\mathbf{x}_{i}, \cdot\right)\left(\mathbf{G}_{X}+n \varepsilon \mathbf{l}_{n}\right)^{-1} \mathbf{Y},
\end{aligned}
$$

where the elements of $\mathbf{G}_{X}$ are $\mathbf{G}_{i j}=K\left(\mathbf{x}_{i}, \mathbf{x}_{j}\right)$. Note that Eq. (8) and Eq. (19b) are equivalent and that $\mathbf{H}=\left(\mathbf{G}_{X}+n \boldsymbol{\varepsilon} \mathbf{I}_{n}\right)^{-1} \mathbf{Y}$.

Different kernel functions $K(\cdot, \cdot)$ describe different nonlinear feature spaces (see examples in appendix A). For the Gaussian kernel function, the columns of $\mathbf{G}_{X}$ [i.e., $K\left(\cdot, \mathbf{x}_{j}\right)$ ] each contain a Gaussian function, centered on $\mathbf{x}_{j}$ and spread over all the other points. Equation (19a) states the function $g(\cdot)$ is a linear combination of those Gaussians. Equation (19b) states that $\nabla g(\cdot)$ is a linear combination of the gradients of those Gaussians.

\section{d. Modeling $f_{j}\left(\mathbf{X W}_{j}\right)$ and estimating $\mathbf{A}$}

Section $2 \mathrm{~b}$ showed how to estimate the matrix $\mathbf{W}$, which contains the unit directions that maximize $\nabla f_{j}\left(\mathbf{X} \mathbf{W}_{j}\right)$ given that $g(\mathbf{X})=\boldsymbol{\Sigma}_{j} f_{j}\left(\mathbf{X} \mathbf{W}_{j}\right)\left(\mathbf{P}_{j}^{Y}\right)^{\prime}$. The directions $\mathbf{W}$ were obtained by modeling $\nabla g(\cdot)$ using a kernel function [Eqs. (8) and (12)], but this only provides the directions $\mathbf{W}$ and not the functions $f_{j}\left(\mathbf{X W}_{j}\right)$ that need to be modeled separately. In addition, in the example in section $2 b, \mathbf{Y}$ was a vector, and so it was not necessary to find a corresponding subspace YA. This subsection shows how to both model $f_{j}\left(\mathbf{X W}_{j}\right)$ and estimate $\mathbf{A}$ in a single step, by combining CCA with basis functions.

For linear CCA, it is well known that the projection matrices $\mathbf{W}^{\mathrm{CCA}}=\mathbf{\Sigma}_{X}^{-0.5} \mathbf{V}_{X}$ and $\mathbf{A}^{\mathrm{CCA}}=\boldsymbol{\Sigma}_{Y}^{-0.5} \mathbf{V}_{Y}$ can be found via two eigenvalue problems:

$$
\begin{aligned}
& \boldsymbol{\Sigma}_{X}^{-0.5} \boldsymbol{\Sigma}_{\hat{X}_{M}} \boldsymbol{\Sigma}_{X}^{-0.5}=\mathbf{V}_{X} \mathbf{L}_{X} \mathbf{V}_{X}^{\prime} \text {, and } \\
& \boldsymbol{\Sigma}_{Y}^{-0.5} \boldsymbol{\Sigma}_{\hat{Y}_{M}} \boldsymbol{\Sigma}_{Y}^{-0.5}=\mathbf{V}_{Y} \mathbf{L}_{Y} \mathbf{V}_{Y}^{\prime},
\end{aligned}
$$

where $\boldsymbol{\Sigma}_{\hat{X}_{M}}$ and $\boldsymbol{\Sigma}_{\hat{Y}_{M}}$ are the covariance matrices of fields $\hat{\mathbf{X}}_{M}$ and $\hat{\mathbf{Y}}_{M}$ estimated using a specific model. For linear CCA, for example $\hat{\mathbf{Y}}_{M}$ is estimated using the linear model [Eq. (13)], so $\boldsymbol{\Sigma}_{\hat{Y}_{M}}=\boldsymbol{\Sigma}_{Y X} \boldsymbol{\Sigma}_{X}^{-1} \boldsymbol{\Sigma}_{X Y}$ [if $\lambda=0$ in Eq. (13)].

For the model in Eq. (4), exactly the same formulation as in Eq. (20b) can be used to estimate $\mathbf{A}$, by using a nonlinear expansion of $\mathbf{X W}$ to calculate $\hat{\mathbf{Y}}_{\mathbf{M}}$. To do this, it is assumed that $f_{j}\left(\mathbf{X} \mathbf{W}_{j}\right)$ can be approximated using a set of basis functions $\phi_{m}(\cdot)$ :

$$
f_{j}\left(\mathbf{X W}_{j}\right)=\sum_{m=1}^{\mathrm{df}} \beta_{m, j} \phi_{m}\left(\mathbf{X} \mathbf{W}_{j}\right),
$$

where $\mathrm{df}$ is the number of basis functions.

There are several possible basis functions that would be suitable here including piecewise linear (appendix B), piecewise polynomial, or orthogonal polynomial basis functions. For any particular application, the parameter df should be chosen according to the degree of nonlinearity that the researcher is interested in. There is an example below, which shows that the basis functions need only to broadly approximate $f_{j}\left(\mathbf{X} \mathbf{W}_{j}\right)$ in order to successfully estimate the directions in $\mathbf{A}$.

For gKCCA, the estimation of $\mathbf{A}$ consists of expanding each $\mathbf{R}_{j}=\mathbf{X} \mathbf{W}_{j}$ by the basis functions $\phi_{m}(\cdot)$ to produce a basis matrix $\phi\left(\mathbf{R}_{j}\right)$ :

$$
\phi\left(\mathbf{R}_{j}\right)=\left[\begin{array}{lll}
\phi_{1}\left(\mathbf{R}_{j}\right) & \cdots & \phi_{d f}\left(\mathbf{R}_{j}\right)
\end{array}\right]
$$

The term $\boldsymbol{\Sigma}_{\hat{Y}_{M}}$ is then estimated as

$$
\boldsymbol{\Sigma}_{\hat{Y}_{M}}=\Sigma_{Y \phi\left(R_{j}\right)} \Sigma_{\phi\left(R_{j}\right)}^{-1} \Sigma_{\phi\left(R_{j}\right) Y}
$$

and substituted into Eq. (20b). Then $\mathbf{A}_{j}=\boldsymbol{\Sigma}_{Y}^{-0.5} \mathbf{V}_{1}$, where $\mathbf{V}_{1}$ is the leading eigenvector of $\mathbf{V}_{Y}$, for each basis matrix $\phi\left(\mathbf{R}_{j}\right)$. This ensures that the columns of $\mathbf{A}$ are paired with the columns of $\mathbf{W}$.

Here the example in section $2 b$ is extended as follows:

$$
\begin{aligned}
\mathbf{Y} & =\mathbf{Y}^{*} \boldsymbol{\Sigma}_{Y^{*}}^{-0.5} \mathbf{A}^{-1}, \quad \text { and } \\
\mathbf{Y}^{*} & =\left[6.3 \exp \left(-0.1 \mathbf{X} \mathbf{W}_{1}\right)\left(\mathbf{X} \mathbf{W}_{1}\right)^{2} \quad \mathscr{N}(0,1)\right]
\end{aligned}
$$

where $\mathbf{Y}$ and $\mathbf{Y}^{*}$ are both $n \times 2$ matrices, $\mathscr{N}(\mu, \sigma)$ denotes numbers sampled from a normal random distribution, and $\mathbf{A}$ is a $2 \times 2$ orthonormal matrix. The matrices $\mathbf{X}$ and $\mathbf{W}$ are the same as in the example in section $2 \mathrm{~b}$. Given only $\mathbf{X}$ and the new $\mathbf{Y}, \mathbf{W}$ was estimated 

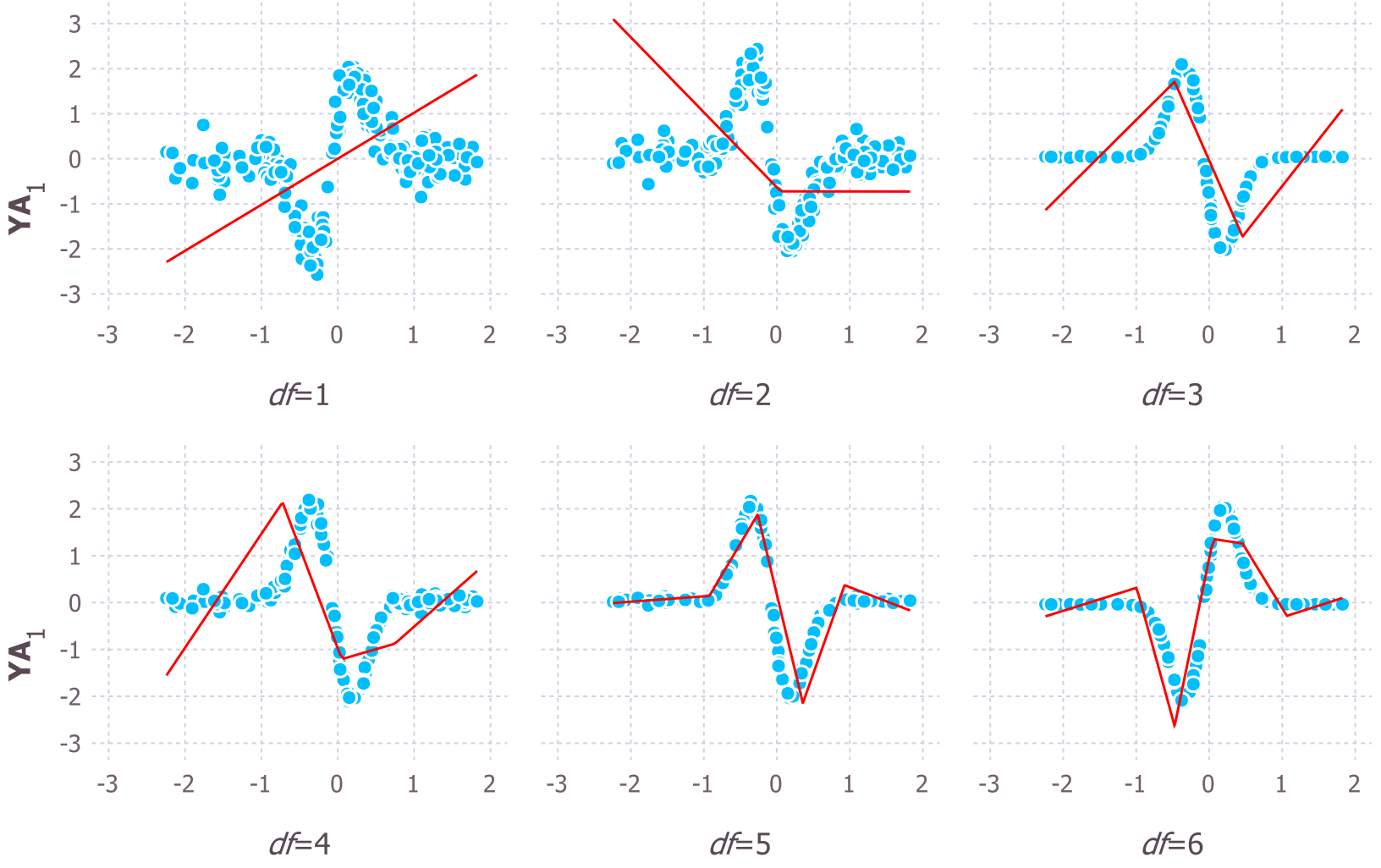

$\mathbf{X} \mathbf{W}_{1}$ (gKCCA)

FIG. 2. The gKCCA method applied to a second simulated example [Eq. (24)]. The plots show the leading component pairs $\mathbf{X W}_{1}$ and $\mathbf{Y A}_{1}$ (blue dots) for gKCCA, estimated using six different values of df. Also shown is the estimated piecewise linear model $\phi\left(\mathbf{R}_{1}\right) \boldsymbol{\Sigma}_{\phi\left(R_{1}\right)}^{-0.5} \mathbf{V}_{X}$ (red line), which "guides" the estimation of $\mathbf{Y A}_{1}$. Note that the number of segments in each piecewise linear model is df, and the change in segment slope occurs at the breakpoints bp (see appendix B).

using the method in section $2 \mathrm{~b}$. To estimate $\mathbf{A}, \phi\left(\mathbf{X W}_{1}\right)$ was calculated using piecewise linear basis functions (appendix B). Piecewise linear basis functions have at least two advantages: they are computationally simple, and the df, the degrees of freedom of or the number of segments in, the piecewise linear basis matrix can be used to control the linearity (or nonlinearity) of the solution.

Figure 2 shows $\mathbf{Y} \mathbf{A}_{1}$ plotted against $\mathbf{X} \mathbf{W}_{1}$ for six different values of df. Also plotted against $\mathbf{X} \mathbf{W}_{1}$ is the piecewise linear model (PWLM), which "guides" the estimation of $\mathbf{Y A}_{1}$, for each df. This piecewise linear model is in fact $\phi\left(\mathbf{R}_{1}\right) \boldsymbol{\Sigma}_{\phi\left(R_{1}\right)}^{-0.5} \mathbf{V}_{X}$ from the application of CCA to $\phi\left(\mathbf{R}_{1}\right)$ and $\mathbf{Y}$. Figure 2 shows that if the PWLM is even a rough approximation of $f_{1}\left(\mathbf{X} \mathbf{W}_{1}\right)$ (e.g., for $\mathrm{df}=3$ ), then the corresponding subspace in $\mathbf{Y}$ that is correlated with $f_{1}\left(\mathbf{X W}_{1}\right)$ can be correctly estimated. For all df $\geq 3$, the PWLM also finds the expected $\mathbf{Y} \mathbf{A}_{1}$. The difference between linear CCA and gKCCA is that linear CCA assumes that $\mathbf{Y \mathbf { A } _ { j }}$ is linearly correlated with $\mathbf{X} \mathbf{W}_{j}$, but gKCCA allows $\mathbf{Y A}_{j}$ to be correlated with $f_{j}\left(\mathbf{X} \mathbf{W}_{j}\right)$. Supplementary section 4 discusses symmetry in CCA and gKCCA.

\section{e. Explained variance and prediction}

Once $\mathbf{W}$ and $\mathbf{A}$ have been estimated, the proportion of the total variance of each field explained by each component can be calculated as follows (as in linear CCA):

$$
\begin{aligned}
& R_{X}^{2}=\operatorname{tr}\left[\boldsymbol{\Sigma}_{X} \mathbf{W}_{j} \mathbf{W}_{j}^{\prime} \boldsymbol{\Sigma}_{X}\right] / \operatorname{tr}\left[\boldsymbol{\Sigma}_{X}\right], \quad \text { and } \\
& R_{Y}^{2}=\operatorname{tr}\left[\boldsymbol{\Sigma}_{Y} \mathbf{A}_{j} \mathbf{A}_{j}^{\prime} \boldsymbol{\Sigma}_{Y}\right] / \operatorname{tr}\left[\boldsymbol{\Sigma}_{Y}\right] .
\end{aligned}
$$

Also, once $\mathbf{W}$ and $\mathbf{A}$ have been estimated it is easy to apply any nonlinear smoothing regression model to the marginal functions $\mathbf{Y} \mathbf{A}_{j}=f_{j}\left(\mathbf{X} \mathbf{W}_{j}\right)$ to build a predictive model for gKCCA.

\section{Cross validation}

\section{Cross-validation metrics for linear and nonlinear models}

The gKCCA method has two main parameters that need to be estimated: $\sigma$ [Eq. (10) in Eq. (19b)] and $\varepsilon$ [Eq. (19b)], for a given df. 
Various implementations of linear CCA contain parameters that need to be estimated; for example, regularized CCA adds ridge parameters to the covariance matrices of the predictor and response fields (Lim et al. 2012). Such parameters are typically estimated using cross validation. The main step of cross validation is, for a particular CCA model with a given set of parameters, to construct the matrices $\mathbf{R}^{\mathrm{cv}}$ and $\mathbf{T}^{\mathrm{cv}}$ using a number of training and test sets:

$$
\begin{aligned}
& \mathbf{R}^{\mathrm{cv}}=\left[\begin{array}{c}
\left(\mathbf{X}^{(1)}-\overline{\mathbf{X}}^{(-1)}\right) \hat{\mathbf{W}}^{(-1)} \\
\vdots \\
\left(\mathbf{X}^{(g)}-\overline{\mathbf{X}}^{(-g)}\right) \hat{\mathbf{W}}^{(-g)}
\end{array}\right], \quad \text { and } \\
& \mathbf{T}^{\mathrm{cv}}=\left[\begin{array}{c}
\left(\mathbf{Y}^{(1)}-\overline{\mathbf{Y}}^{(-1)}\right) \hat{\mathbf{A}}^{(-1)} \\
\vdots \\
\left(\mathbf{Y}^{(g)}-\overline{\mathbf{Y}}^{(-g)}\right) \hat{\mathbf{A}}^{(-g)}
\end{array}\right],
\end{aligned}
$$

where $\mathbf{X}^{(1)}, \ldots, \mathbf{X}^{(g)}$ refer to the test sets and $\mathbf{X}^{(-1)}, \ldots, \mathbf{X}^{(-g)}$ refer to the training sets, $\overline{\mathbf{X}}^{(-g)}$ refers to a matrix of column means, and $\mathbf{W}^{(-g)}$ refers to the projection matrix calculated for the respective training set. The skill of a particular CCA model can then be assessed using a cross-validation metric, such as a measure of the linear association between the subspaces $\mathbf{R}^{\mathrm{cv}}$ and $\mathbf{T}^{\mathrm{cv}}$ (e.g., Wilk's lambda, Hotelling-Lawley trace, or the Pillai-Bartlett trace). These metrics can be calculated on a selected number of dimensions of $\mathbf{R}^{\mathrm{cv}}$ and $\mathbf{T}^{\mathrm{cv}}$, and this number of dimensions is referred to as $d_{\text {cv }}$. Note that $d_{\mathrm{cv}}$ is required only for the calculation of the crossvalidation metric and is separate from $d$ [e.g., Eq. (4)], which is the number of model dimensions. That is, $d_{\mathrm{cv}}$ is a metric parameter and $d$ is a model parameter. It is possible that $d>d_{\mathrm{cv}}$ or that $d<d_{\mathrm{cv}}$. For example, we could cross-validate a CCA model using only the first dimension $\mathbf{R}_{1}^{\mathrm{cv}}$ and $\mathbf{T}_{1}^{\mathrm{cv}}$ (i.e., $d_{\mathrm{cv}}=1$ ) but could later find that there is a meaningful association between $\mathbf{X} \mathbf{W}_{2}$ and $\mathbf{Y A}_{2}(d=2)$, after calculating the full CCA model using the cross-validated parameters. Since $d_{\mathrm{cv}}$ is a metric parameter (and not a model parameter), the choice of $d_{\mathrm{cv}}$ should be made in the same way as the choice of metric (e.g., Wilk's lambda, Hotelling-Lawley trace, and Pillai-Bartlett trace are metrics of linear association): the choice should be based on the user's intuition about what they are attempting to solve.

For gKCCA, measures of linear association between $\mathbf{R}^{\mathrm{cv}}$ and $\mathbf{T}^{\mathrm{cv}}$ are not sufficient because $\mathbf{R}^{\mathrm{cv}}$ and $\mathbf{T}^{\mathrm{cv}}$ may be nonlinearly dependent. Various measures of nonlinear association between subspaces have been suggested including entropy-based and distancecorrelation-based statistics (e.g., Martínez-Gómez et al. 2014). One difficulty with these statistics is that they measure the nonlinear association between the total subspaces of $\mathbf{R}_{1: d_{\mathrm{cv}}}^{\mathrm{cv}}$ and $\mathbf{T}_{1: d_{\mathrm{cv}}}^{\mathrm{cv}}$ and thus are independent of the rotation of any particular subspace (e.g., $\left.\mathbf{T}_{1: d_{\mathrm{cv}}}^{\mathrm{cv}}\right)$. The aim here is to use a metric that captures the nonlinear association between $\mathbf{R}_{j}^{\mathrm{cv}}$ and $\mathbf{T}_{j}^{\mathrm{cv}}$, for each $j=\left(1, \ldots, d_{\mathrm{cv}}\right)$. This can be achieved by expanding each $\mathbf{R}_{j}^{\text {cv }}$ using a set of orthogonal basis functions, as in section $2 \mathrm{~d}$. The parameter $\mathrm{df}$ is again the number of basis functions.

The actual nonlinear cross-validation metric is the adjusted multiple coefficient of determination $R^{2}$ (also known as the squared multiple correlation coefficient) from an augmented linear model (ALM):

$$
\begin{aligned}
\left(\begin{array}{c}
\mathbf{T}_{1}^{\mathrm{cv}} \\
\vdots \\
\mathbf{T}_{d_{\mathrm{cv}}}^{\mathrm{cv}}
\end{array}\right) & =\left[\begin{array}{ccc}
\phi\left(\mathbf{R}_{1}^{\mathrm{cv}}\right) & \mathbf{0} & \mathbf{0} \\
\mathbf{0} & \ddots & \mathbf{0} \\
\mathbf{0} & \mathbf{0} & \phi\left(\mathbf{R}_{d_{\mathrm{cv}}}^{\mathrm{cv}}\right)
\end{array}\right] \mathbf{B}^{\mathrm{cv}}+\left(\begin{array}{c}
\mathbf{E}_{1}^{\mathrm{cv}} \\
\vdots \\
\mathbf{E}_{d_{\mathrm{cv}}}^{\mathrm{cv}}
\end{array}\right), \quad \text { and } \\
R_{\mathrm{cv}}^{2} & =1-\frac{\mathrm{SS}_{E^{\mathrm{cv}}}}{\left[d_{\mathrm{cv}}(n-\mathrm{df})-1\right]} \frac{\left(d_{\mathrm{cv}} n-1\right)}{\mathrm{SS}_{T^{\mathrm{cv}}}},
\end{aligned}
$$

where $\mathbf{B}^{\mathrm{cv}}$ is a $\left(d_{\mathrm{cv}} \cdot \mathrm{df}\right)$-length vector of regression coefficients, and $\mathrm{SS}_{E}$ is the sum of the squares of the subscripted vector (or matrix). For the ALM, the response variable [left-hand side of Eq. (27a)] is formed by vectorizing the columns of $\mathbf{T}^{\mathrm{cv}}$, while the matrices $\phi\left(\mathbf{R}_{j}^{\mathrm{cv}}\right)$ are basis function matrices created for the $j^{\text {th }}$ column of $\mathbf{R}^{\mathrm{cv}}$ [Eq. (22)]. Since the response variable of this ALM is a vector, the adjusted multiple coefficient of determination $R_{\mathrm{cv}}^{2}$ is a scalar value [Eq. (27b)]. Note the adjusted multiple correlation coefficient is different from the simple multiple correlation coefficient: the adjusted coefficient takes into account the degrees of freedom of the ALM.

\section{Nonlinearly coupled oscillations in the Pacific Ocean}

\section{a. Introduction}

In this section, gKCCA is used to investigate nonlinear coupling in the Pacific Ocean.

It is likely that ENSO has multiple modes of variability, each with their own characteristic time scale, that can coexist (Barnett 1991). This aspect of ENSO has been investigated by several researchers using the Zebiak-Cane (ZC) model (Yang et al. 1997; Roulston and Neelin 2003; Bejarano and Jin 2008). For example, Bejarano and Jin (2008) ran the full ZC model over a wide range of values of a two-dimensional parameter space, the parameters being the upper-ocean layer 
thickness and the percentage wind stress. The ZC model exhibited three main regimes:

1) A quasi-quadriennial regime, which dominated for deep upper-layer thickness $(150 \mathrm{~m})$ and high-scaled wind stress $(>1)$

2) A quasi-biennial $\mathrm{QB}$ regime, which dominated for shallow upper-layer thickness $(130 \mathrm{~m})$ and highscaled wind stress $(>1)$

3) A mixed regime, where the $Q Q$ and $Q B$ modes coexisted, for average upper-layer thickness $(140 \mathrm{~m})$ and average scaled wind stress $(=1)$

Thus Bejarano and Jin (2008) demonstrated that ENSO may consist of two oscillatory modes, which can coexist, but their study did not examine the interaction between the modes.

Earlier work on the interaction between the QQ and QB modes was conducted by Barnett (1991), Yang et al. (1997), and Roulston and Neelin (2003). Using bicoherence to analyze global SST data (1950-87), Barnett (1991) showed that the QQ and QB modes were quadratically coupled, while the QB mode and the annual mode were not quadratically coupled. Yang et al. (1997) and Roulston and Neelin (2003) performed experiments with a linearized version and a lite nonlinear version of the ZC model, respectively. In the full ZC model, SST is a nonlinear function of thermocline depth, and atmospheric heating is a nonlinear function of surfacewind convergence, but in the Roulston and Neelin model only the former function is nonlinear (hence lite nonlinear version). Both Yang et al. (1997) and Roulston and Neelin (2003) performed their model experiments without a seasonal cycle. Yang et al. (1997) found that the most unstable mode was a quasi-biennial oscillation, and the linearized model produced no quasiquadrennial oscillation, suggesting that the QQ mode might arise from a nonlinear interaction with the QB mode. In contrast, Roulston and Neelin (2003) found that the QB mode was stable and only became oscillatory as a result of nonlinear coupling to an unstable QQ mode. Also, the nonlinearity in their model did not produce a quasi-biennial spectral peak without the presence of a stable separate QB mode. That is, the QB spectral peak was not simply a subharmonic of the QQ mode.

In another study, Monahan and Dai (2004) considered the following statistical model, which is rewritten here in terms of the notation used in this manuscript:

$$
\hat{\mathbf{X}}=\mathbf{X} \mathbf{W}_{1} \mathbf{P}_{1}^{\prime}+\left(\mathbf{X} \mathbf{W}_{1}\right)^{2} \mathbf{P}_{2}^{\prime}+\mathbf{1}_{n} \mathbf{P}_{3}^{\prime}
$$

where here $\mathbf{X}$ is the tropical Pacific SST anomaly field, and $\mathbf{P}$ is a $p \times 3$ matrix of spatial patterns. This model basically says that there are two coupled modes in the tropical Pacific, with spatial patterns $\mathbf{P}_{1}$ and $\mathbf{P}_{2}$ and time components $\mathbf{X} \mathbf{W}_{1}$ and $\left(\mathbf{X} \mathbf{W}_{1}\right)^{2}$, which Monahan and Dai (2004) refer to as the antisymmetric component and the symmetric component, respectively. Monahan and Dai (2004) fitted this model by assuming that $\mathbf{W}_{1}$ was the first EOF of $\mathbf{X}$, and then $\mathbf{P}$ was determined using standard linear techniques. In this model, since the time component of the second mode peaks twice as fast as the first mode, in unison with the extreme positive and negative anomalies of the first mode, the two modes represented by spatial patterns $\mathbf{P}_{1}$ and $\mathbf{P}_{2}$ are nonlinearly coupled. Monahan and Dai (2004) found that this model explained some variability in the observed SST field, but the same coupling was generally absent in modeled SST fields from global climate models, and hence the mechanisms of mode coupling were not investigated.

\section{b. Data and analysis}

The main data used in this study are from the Extended Reconstructed Sea Surface Temperature (ERSST) dataset (Huang et al. 2016; NOAA 2017), which spans the years 1854 to present and has a spatial resolution of $2^{\circ} \times 2^{\circ}$. Monthly data were extracted for the region $125^{\circ}$ to $295^{\circ} \mathrm{E}$ and $-20^{\circ}$ to $20^{\circ} \mathrm{N}(p=q=1555)$, for the years 1945-2009 $(n=780)$. To minimize edge effects on the bandpass filtering (see below), the raw time series (1854-2016) were first filtered, and then the years 1945-2009 were obtained from the filtered fields. The aim here is to investigate the nature of the coupling between QB and QQ components of the observational SST field, so the SST field was separated into a predictor field and a response field by using two Butterworth bandpass filters of order 3 . The predictor field $\mathbf{X}$ was produced by using a 4-6-yr bandpass filter. The response field $\mathbf{Y}$ was produced by using a 2-3-yr bandpass filter. Henceforth, the predictor field and response field are also referred to as the low-pass field and the high-pass field, respectively. This is a good example for illustrating the gKCCA method because we would not expect any linear correlation between two fields containing orthogonal frequencies. Three preprocessing steps were applied prior to analysis: the $\mathbf{X}$ and $\mathbf{Y}$ fields were centered and scaled to unit variance, then weighted by the square root of cos(latitude), and whitened using singular value decomposition (Chung and Nigam 1999). In the latter step, because $p$ and $q$ were $\gg n$, only the components that collectively explained up to $85 \%$ of the total field variance were kept. The gKCCA method was also applied to predictor and response fields, which retained $80 \%$ and $90 \%$ of the total field variance, but in both cases the cross-validation metric $R_{\mathrm{cv}}^{2}$ was less than when 
$85 \%$ of the total field variance was retained (see section 4c).

The coupling between the $\mathbf{X}$ and $\mathbf{Y}$ fields was investigated using both regularized linear CCA (regCCA) and gKCCA. For regCCA, the covariance matrix of each field was regularized, and the regCCA model was estimated using singular value decomposition:

$$
\left(\boldsymbol{\Sigma}_{X}+\lambda_{X} \mathbf{l}_{p}\right)^{-0.5} \boldsymbol{\Sigma}_{X Y}\left(\boldsymbol{\Sigma}_{Y}+\lambda_{Y} \mathbf{l}_{q}\right)^{-0.5}=\mathbf{v}_{X} \mathbf{D} \mathbf{V}_{Y}^{\prime},
$$

where $\mathbf{V}_{X}$ and $\mathbf{V}_{Y}$ are the projection matrices for the whitened $\mathbf{X}$ and $\mathbf{Y}$ fields. Thus the regCCA model has two parameters to be estimated: $\lambda_{X}$ and $\lambda_{Y}$. Similarly, the gKCCA model has two parameters to be estimated: $\varepsilon$ and $\sigma$.

For both regCCA and gKCCA the model parameters were estimated using gridded parameter values and leave-half-out cross-validation, with the cross-validation metric $R_{\mathrm{cv}}^{2}$. For regCCA, a grid of six values for both $\lambda_{X}$ and $\lambda_{Y}$ were used: $\lambda=10^{l}(-7 \leq l \leq-2)$. These values were also used for $\varepsilon$ in gKCCA. For $\sigma$ in gKCCA, 10 different values were used: $\sigma=c \sigma_{\text {med }}(0.15 \leq c \leq 2)$, where $\sigma_{\text {med }}$ is the median of pairwise Euclidean distances of the data [Eq. (11)]. Also for gKCCA, piecewise linear basis functions were used in Eq. (23) and Eq. (27).

In addition, the two other parameters $\mathrm{df}$ and $d_{\mathrm{cv}}$ were set based on the problem at hand here. For regCCA and gKCCA, the parameter df [in Eq. (27)] was set at df $=1$ and $\mathrm{df}=2$ respectively, which means that the crossvalidation metric $R_{\mathrm{cv}}^{2}$ provides information about linear $(\mathrm{df}=1)$ and piecewise linear $(\mathrm{df}=2)$ association. For gKCCA, the rationale for setting $\mathrm{df}=2$ was that it is potentially quadratic (or $\mathrm{V}$ shaped) interactions that are of interest here. For both regCCA and gKCCA, the metric parameter $d_{\mathrm{cv}}$ was set at $d_{\mathrm{cv}}=1$ so that $R_{\mathrm{cv}}^{2}$ is a measure of association between $\mathbf{R}_{1}^{\mathrm{cv}}$ and $\mathbf{T}_{1}^{\mathrm{cv}}$. The rationale for setting $d_{\mathrm{cv}}=1$ was that a preliminary analysis suggested that there were no statistically significant subspaces beyond the first gKCCA subspace. Considering df and $d_{\mathrm{cv}}$ together, for regCCA, $R_{\mathrm{cv}}^{2}$ is a measure of linear correlation between $\mathbf{R}_{1}^{\mathrm{cv}}$ and $\mathbf{T}_{1}^{\mathrm{cv}}$ (because $\mathrm{df}=1$ and $\left.d_{\mathrm{cv}}=1\right)$. For gKCCA, $R_{\mathrm{cv}}^{2}$ is a measure of nonlinear (or piecewise linear) correlation between $\mathbf{R}_{1}^{\mathrm{cv}}$ and $\mathbf{T}_{1}^{\mathrm{cv}}$ (because $d f=2$ and $d_{\mathrm{cv}}=1$ ).

Further for gKCCA, after cross-validating the parameters $\sigma$ and $\varepsilon$, a multivariate phase randomization test (Schreiber and Schmitz 2000) was performed to test if the coupling between the components $\mathbf{R}_{j}$ and $\mathbf{T}_{j}$ was statistically significant. In the phase randomization test, null hypothesis surrogates were generated by randomizing the Fourier phases of the predictor and response fields. This means that the time series in the randomized fields have the same Fourier spectra as the time series in the predictor and response fields, but with different phases. A thousand surrogate pairs of fields were generated, whitened, and gKCCA was applied using the cross-validated estimates of the parameters $\sigma$ and $\varepsilon$. The nonlinear coupling between fields was tested using a time-domain version of the bicoherence statistic $b$ :

$$
b_{j}=\operatorname{cor}\left(\mathbf{R}_{j} \odot \mathbf{R}_{j}, \mathbf{T}_{j}\right)
$$

where $\odot$ denotes the Hadamard product. Here the bicoherence is a linear correlation between $\mathbf{R}_{j} \odot \mathbf{R}_{j}$ and $\mathbf{T}_{j}$, or a measure of the quadratic phase coupling between the dominant frequencies in $\mathbf{R}_{j}$ and $\mathbf{T}_{j}$. The multivariate phase randomization test is essentially asking how likely it is that the nonlinear coupling between the observed fields could be the result of nonlinear alignment between random fields that oscillate at the same frequencies as the observed fields. This test is more general than the test performed by Barnett (1991), which modeled the leading field components as a second-order autoregressive process. The multivariate phase randomization test contains no assumption about the order of the autoregressive process.

\section{c. Results}

For regCCA, the cross-validated parameter estimates were $\lambda_{X}=10^{-7}$ and $\lambda_{Y}=10^{-7}$. For gKCCA, the crossvalidated parameter estimates were $\sigma=1.6 \sigma_{\text {med }}$ and $\varepsilon=10^{-3}$. The term $R_{\mathrm{cv}}^{2}$ was higher for gKCCA $(0.23)$ than for regCCA $(\sim 0.00)$, suggesting that there is no linear coupling between $\mathbf{X}$ and $\mathbf{Y}$. Note that for gKCCA, $R_{\mathrm{cv}}^{2}$ was $0.21,0.23$, and 0.14 when retaining $80 \%, 85 \%$, and $90 \%$ of the total field variance. The respective dimensionality of $\mathbf{X}$ was 3,3 , and 4 components (for $80 \%$, $85 \%$, and $90 \%$ of the field variance), while the dimensionality of $\mathbf{Y}$ was 4,6 , and 8 components. Thus in this example, the main decrease in $R_{\mathrm{cv}}^{2}$ is associated with the increasing dimensionality of $\mathbf{Y}$ and consequent overfitting of the model in the leave-half-out cross-validation. The generally low $R_{\mathrm{cv}}^{2}$ values suggest that the nonlinear coupling between the high-pass and low-pass fields is not particularly strong.

Figure 3 shows the correlation between the paired components $\mathbf{X W}_{j}$ and $\mathbf{Y} \mathbf{A}_{j}$, and the associated spatial patterns, for the leading two regCCA subspaces. As expected, the linear correlations here are not significant (Fig. 3, left column), so there is no interaction between the spatial patterns.

Table 2 contains the results of the multivariate phase randomization test applied to the leading three gKCCA subspaces. The bicoherence of the first component pair is significantly different from the null hypothesis bicoherence distribution, while the bicoherences of the 


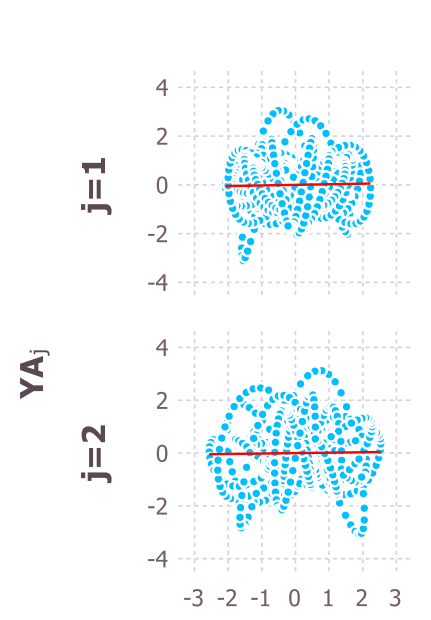

$\mathbf{X} \mathbf{W}_{\mathrm{j}}$

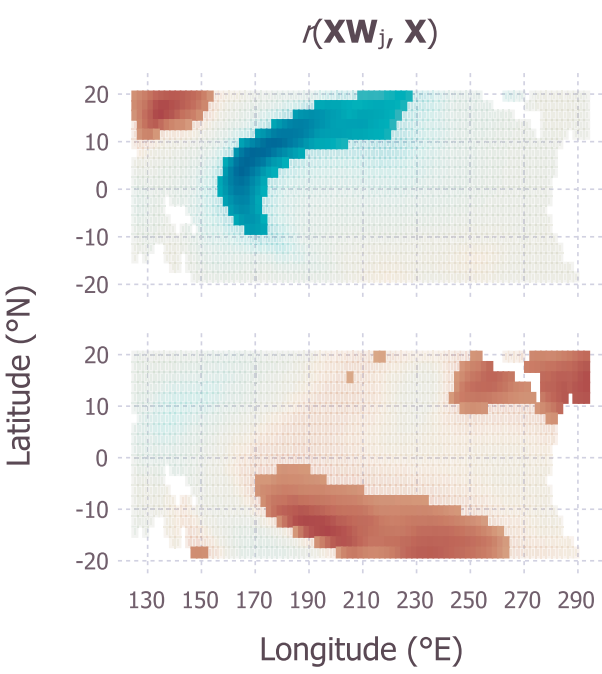

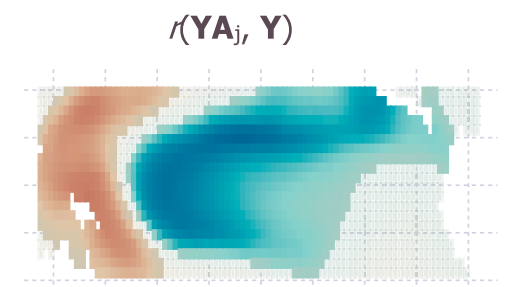
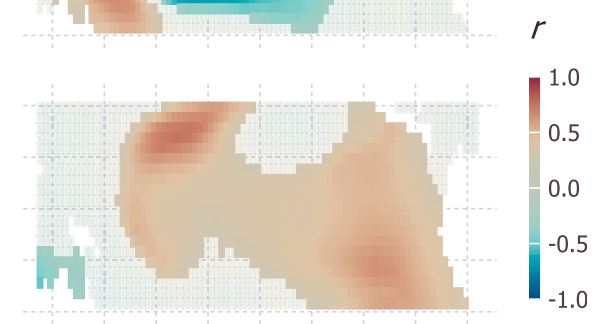

130150170190210230250270290 Longitude $\left({ }^{\circ} \mathrm{E}\right)$

FIG. 3. The first two leading component pairs $\mathbf{X} \mathbf{W}_{j}$ and $\mathbf{Y} \mathbf{A}_{j}$ using canonical correlation analysis, where $\mathbf{X}$ is the low-pass SST field, and $\mathbf{Y}$ is the high-pass SST field. Each row corresponds to a different $j=(1,2)$. (left) The correlation between $\mathbf{X W}$ and $\mathbf{Y} \mathbf{A}_{j}$; (center),(right) the spatial anomaly patterns. The shading corresponds (center) to the correlation (Pearson's $r$ ) between $\mathbf{X W}_{j}$ and the low-pass field and (right) to the correlation between $\mathbf{Y} \mathbf{A}_{j}$ and the high-pass field. Correlations that are not significant (i.e., that fall within the $10 \%-90 \%$ confidence limits of a bivariate red noise hypothesis) have been grayed over. The effective sample size for this test is $n_{\mathrm{eff}}=n\left[\left(1-\rho_{1} \rho_{2}\right) /\left(1+\rho_{1} \rho_{2}\right)\right]$, where $\rho_{1}$ and $\rho_{2}$ are the lag-one autocorrelations of two time series.

second and third subspace are not significantly different. This demonstrates that the quadratic phase coupling between the $\mathbf{X}$ and $\mathbf{Y}$ fields identified in the leading gKCCA subspace is not simply the result of random alignment between two fields that oscillate at the same frequency as $\mathbf{X}$ and $\mathbf{Y}$.

Figure 4 shows the correlation between the paired components $\mathbf{X} \mathbf{W}_{j}$ and $\mathbf{Y} \mathbf{A}_{j}$ and the spatial patterns for the leading two gKCCA subspaces. For $j=1$, the correlation between $\mathbf{X} \mathbf{W}_{j}$ and $\mathbf{Y} \mathbf{A}_{j}$ shows a general quadratic relationship (Fig. 4, top left). Components $\mathbf{X} \mathbf{W}_{1}$ and $\mathbf{Y} \mathbf{A}_{1}$ explain $47 \%$ and $30 \%$ of the variance in the original lowpass and high-pass SST field, respectively (Fig. 4, top row). The positive phase of $\mathbf{X W}_{1}$ shows negative anomalies in the equatorial east and central Pacific, and horseshoe-shaped positive anomalies in the west Pacific. The positive phase of $\mathbf{Y} \mathbf{A}_{1}$ shows positive anomalies in the equatorial central Pacific. Figure 5 (top row) shows that $\mathbf{X} \mathbf{W}_{1}$ and $\mathbf{Y} \mathbf{A}_{1}$ oscillate with periods of 5 and $\sim 2.5$ years respectively. The quadratic relationship between $\mathbf{X} \mathbf{W}_{1}$ and $\mathbf{Y} \mathbf{A}_{1}$ means that positive phases of $\mathbf{Y} \mathbf{A}_{1}$ are associated with both positive and negative phases of $\mathbf{X} \mathbf{W}_{1}$, while negative phases of $\mathbf{Y} \mathbf{A}_{1}$ are associated with neutral phases of $\mathbf{X} \mathbf{W}_{1}$ (Fig. 5, top row). Here the highfrequency mode $\mathbf{Y A}_{1}$ has a stronger pattern in the central Pacific than in the east Pacific (Fig. 4, top right), which contrasts with the high-frequency (symmetric) mode of Monahan and Dai (2004). In Barnett (1991), the high-frequency (QB) mode exhibited a strong pattern in the both the central and east Pacific. In these other studies, the low-frequency and high-frequency patterns (which are nonlinearly related) were estimated in very different ways. Here these patterns have been extracted by maximizing a general measure of association (the squared directional derivative) between two fields. The mechanics of this nonlinear coupling will be investigated in a future study using global climate models.

The second pair of gKCCA components $\mathbf{X} \mathbf{W}_{2}$ and $\mathbf{Y} \mathbf{A}_{2}$ explain $34 \%$ and $7 \%$ of the variance in the original lowpass and high-pass SST field, respectively (Fig. 4, bottom row). The gKCCA method has attempted to find a subspace in which the components $\left(\mathbf{X} \mathbf{W}_{2}\right.$ and $\left.\mathbf{Y} \mathbf{A}_{2}\right)$ are nonlinearly related, but this subspace is not statistically significant (Table 2).

\section{Discussion}

In this section, the gKCCA method is further investigated with respect to the effect of noise on the estimation of the parameters $\sigma$ and $\varepsilon$ and the application

TABLE 2. Bicoherence results from multivariate phase randomization test.

\begin{tabular}{lcc}
\hline & Surrogates $(0 \%-95 \%$ limits $)$ & Observed \\
\hline Subspace 1 & $0.05-0.52$ & 0.56 \\
Subspace 2 & $0.04-0.52$ & 0.39 \\
Subspace 3 & $0.06-0.53$ & 0.43 \\
\hline
\end{tabular}




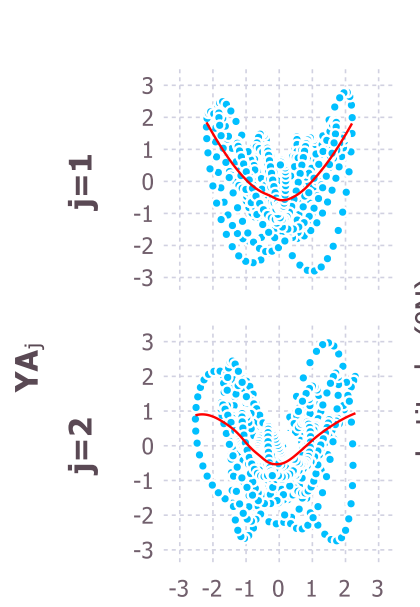

$\mathbf{X} \mathbf{W}_{\mathrm{j}}$
$r\left(\mathbf{X} \mathbf{W}_{\mathrm{j}}, \mathbf{X}\right)$

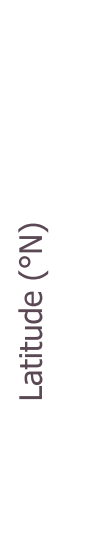

FIG. 4. The first two leading component pairs $\mathbf{X} \mathbf{W}_{j}$ and $\mathbf{Y} \mathbf{A}_{j}$ using gKCCA. Each row corresponds to a different $j=(1,2)$. (left) The correlation between $\mathbf{X} \mathbf{W}_{j}$ and $\mathbf{Y} \mathbf{A}_{i}$; (center),(right) the spatial anomaly patterns. Shading as in Fig. 3. The percentages (in the top right corner of the spatial plots) refer to the proportion of variance that the respective component explains in the total $\mathbf{X}$ and Y fields.

of other kernels. These aspects are explored using a third simulated example.

In this example, a quadratically coupled pair of fields were simulated as follows:

$$
\mathbf{X}_{j}=Z\left[\alpha_{j} \sin \left(\frac{2 \pi t}{5}\right)\right]+\mathscr{N}(0,0.05), \quad \text { and }
$$

$$
\mathbf{Y}_{j}=Z\left[\alpha_{j} \sin \left(\frac{2 \pi t}{2.5}+1.5 \pi\right)\right]+\mathscr{N}\left(0, \sigma_{e}\right)
$$

where $\alpha_{j}=-1$ or 1 (chosen randomly for $\mathbf{X}$ and $\left.\mathbf{Y}\right), t$ is the time in decimal years (with monthly resolution), and $Z[\cdot]$ is the $z$-score operator. Both the $\mathbf{X}$ and $\mathbf{Y}$ fields were simulated with dimensions $780 \times 10$ (i.e., $n \times p$ ). In the two experiments that follow, the gKCCA

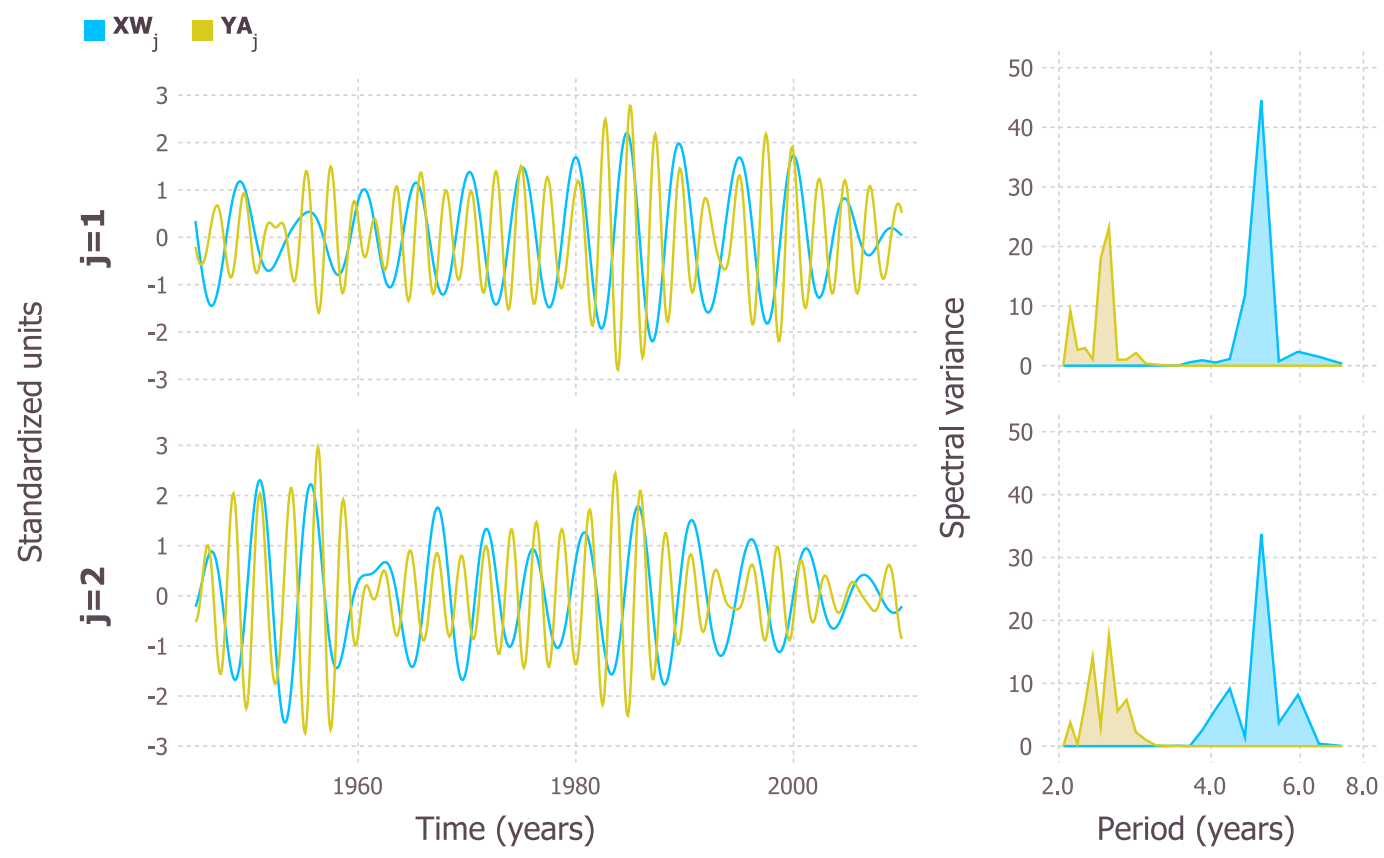

FIG. 5. (left) The gKCCA time components of the low-pass SST field $\mathbf{X W}_{j}$ and the high-pass SST field $\mathbf{Y} \mathbf{A}_{j}$ and (right) their spectra. Each row corresponds to a different $j$. 

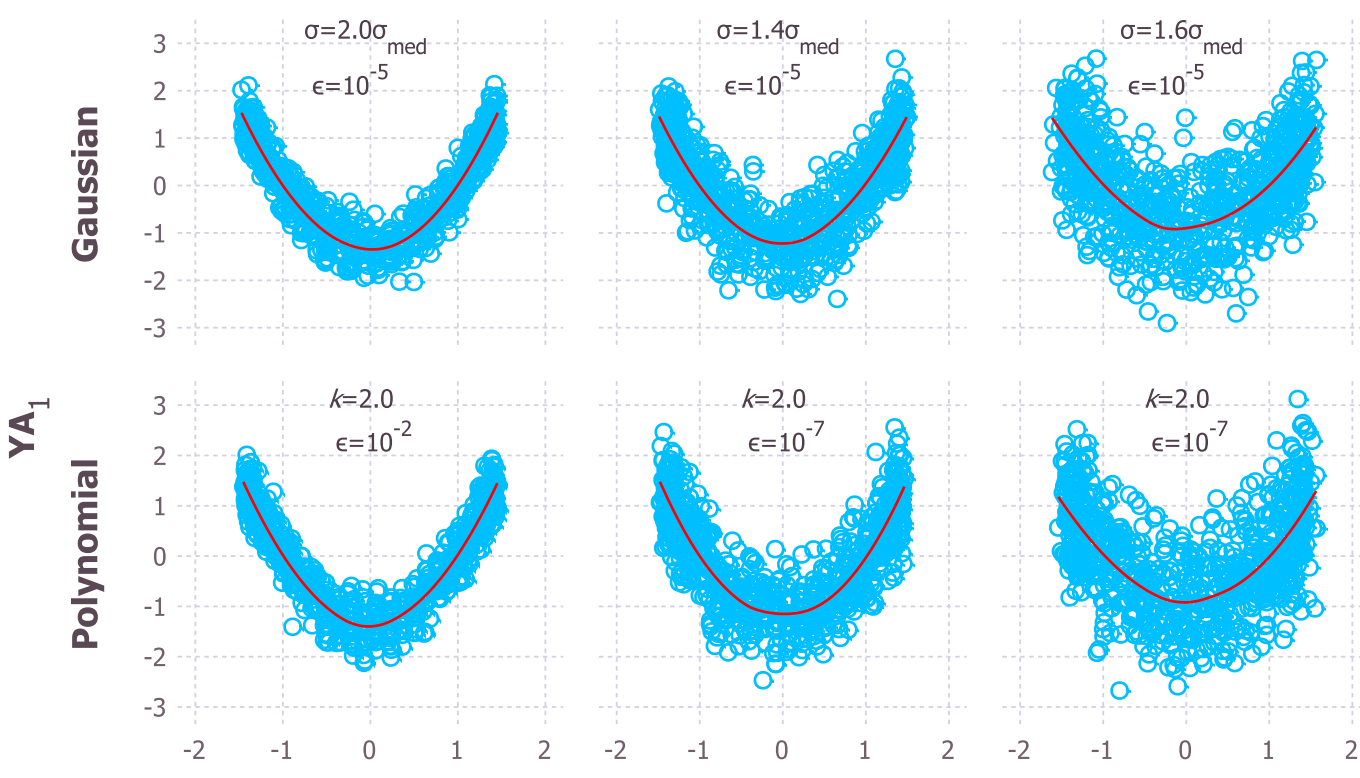

SNR $=1.0$
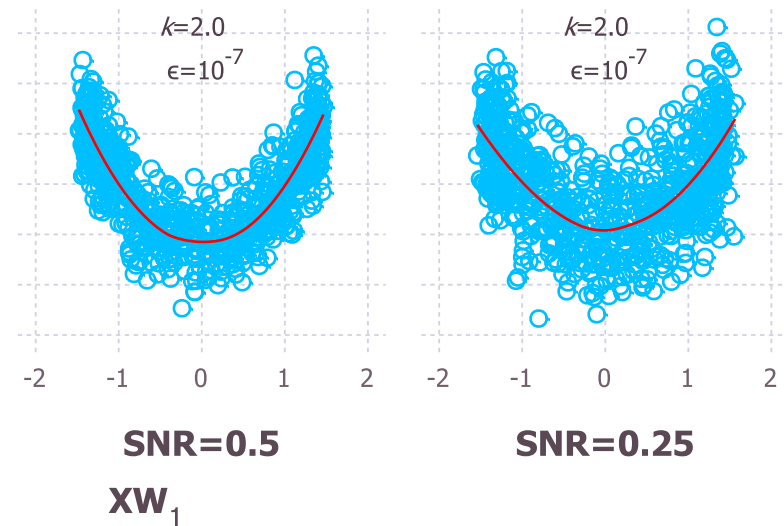

FIG. 6. The sensitivity of gKCCA to different noise levels and kernel functions. The gKCCA method was applied to the third simulated example [Eq. (30)]. Each plot shows the correlation between $\mathbf{X W}_{j}$ and $\mathbf{Y} \mathbf{A}_{j}$ (blue circles) with a loess smooth (red lines). The columns correspond to different levels of noise in $\mathbf{Y}$, and the rows correspond to different kernel functions. On each plot, the cross-validated parameter estimates are given.

method was used to estimate the leading subspace, for three different values of $\sigma_{e}=(1.0,2.0,4.0)$, which corresponds to a signal-to-noise ratio $\mathrm{SNR}=$ $(1.0,0.5,0.25)$ for simulated $\mathbf{Y}$. The gKCCA parameters were estimated using leave-half-out cross validation applied to gridded parameter sets (see below for details).

In the first experiment, the gKCCA method was applied using a Gaussian kernel. The parameter $\sigma_{\text {med }}$, which is calculated for the predictor field $\mathbf{X}$, was the same $\left(\sigma_{\text {med }}=4.3\right)$ for all values of SNR. The parameters $\sigma=c \sigma_{\text {med }}$ and $\varepsilon$ were estimated using gridded parameter values (the same as in section $4 \mathrm{~b}$ ). The leading subspace, for the three different values of SNR, are shown in Fig. 6 (top row): the quadratic relationship between $\mathbf{X} \mathbf{W}_{1}$ and $\mathbf{Y} \mathbf{A}_{1}$ is visible in all simulations. The parameter $\varepsilon$ was the same for all three values of SNR. The parameter $\sigma=c \sigma_{\text {med }}$ did not vary systematically for the different values of SNR, suggesting the different values of $\sigma$ were simply due to the randomness in the simulations. This example suggests that for the Gaussian kernel the parameters $\sigma$ and $\varepsilon$ are insensitive to the level of noise in $\mathbf{Y}$.

In the second experiment, the gKCCA method was applied using a polynomial kernel. Unlike the Gaussian kernel, the feature space of the polynomial kernel is finite dimensional (appendix A). The polynomial kernel and its derivative are

$$
\begin{aligned}
K_{p}\left(\mathbf{x}_{i}, \mathbf{x}_{j}\right) & =\left(\mathbf{x}_{i} \mathbf{x}_{j}^{\prime}+1.0\right)^{k} \quad \text { and } \\
\nabla K_{p}\left(\mathbf{x}_{i}, \mathbf{x}_{j}\right) & =k \mathbf{x}_{j}\left(\mathbf{x}_{i} \mathbf{x}_{j}^{\prime}+1.0\right)^{k-1},
\end{aligned}
$$

where $k$ is the power of the polynomial. Thus for this experiment there are also two parameters to be estimated: $\varepsilon$ [Eq. (19b)] and $k$. The parameter $\varepsilon$ was estimated using the same gridded parameter set as above. The parameter $k$ was estimated using the set $k=(1,2,3)$. Given that the above simulation contains quadratic coupling, a value of $k=2$ is expected. The leading subspace, for the three values of SNR, are shown in Fig. 6 (bottom row). Again, the quadratic relationship between $\mathbf{X} \mathbf{W}_{1}$ and $\mathbf{Y A}_{1}$ is visible in all simulations. The cross-validated parameter $k$ was the same $(k=2)$ for all three values of SNR. The parameter $\varepsilon$ showed values of $\varepsilon=10^{-2}$ and $\varepsilon=10^{-7}$ for values of $\mathrm{SNR}=1.0$ and $\mathrm{SNR} \leq 0.5$, respectively. Each eigenfunction of the polynomial kernel matrix will contain all the monomials of a particular polynomial (this stems from the polynomial feature space; appendix A). This means that quadratic features will be distributed through all the eigenfunctions, and hence heavy 
regularization (a high value of $\varepsilon$ ) is not expected when using the polynomial kernel. In this example, using $\varepsilon=10^{-2}$ or $\varepsilon=10^{-7}$ with the polynomial kernel produces little to no difference in the estimated leading subspace.

The investigation of nonlinearities in climate science has been hindered by the lack of methods which are relatively easy to use and computationally efficient. There are many areas in climate science where gKCCA could be applied, and two further examples are given here. The first is in the area of climate reconstructions using climate proxies, such as tree rings. Tree rings carry a climate signal that is both nonlinear and multivariate (Evans et al. 2014). It would be interesting to test how well gKCCA reconstructs temperature and rainfall variation in the context of pseudoproxy experiments with nonlinear proxy models, such as VS-Lite (VaganovShashkin-Lite), a tree-ring model (Tolwinski-Ward et al. 2011). VS-Lite is an example of a proxy model that defines a nonlinear function between climate variables (temperature, precipitation) and the climate proxy (tree rings). The gKCCA method should be able to find such a nonlinear function. The second example is in the area of predictability. For example, in the Lorenz model, the state variables at one point in time are nonlinearly dependent on the state at past times. This nonlinear predictability has been investigated using a neural network (Cannon 2006), and it would be interesting to see how gKCCA performs. The gKCCA method can also be developed further. For example, it is possible that there could be nonlinear coupling between fields with propagating signals. Complex CCA (i.e., CCA with complex vectors) can be used to investigate linear coupling between such fields (Schreier 2008), so complex gKCCA needs to be developed in order to investigate nonlinear coupling between fields with propagating signals.

\section{Conclusions}

Gradient-based kernel dimension reduction relies on two powerful mathematical ideas: (i) the gradient vector $\nabla g\left(\mathbf{x}_{i}\right)$ in an input space is a linear combination of the gradient vectors $\nabla \Phi\left(\mathbf{x}_{i}\right)$ in a feature space, and (ii) a kernel function can be used to implicitly describe a feature space, without explicitly computing the mapping functions $\Phi\left(\mathbf{x}_{i}\right)$. Two methods that have been previously suggested for investigating nonlinear dependence between two fields are KCCA and NNCCA. KCCA uses the second idea above but only recovers components of the feature space. In contrast, gKCCA uses the feature space to recover components of the input space.

This paper has introduced the gKCCA method and investigated several aspects of the method, including nonlinear cross validation, sensitivity to noise, and the application of different kernel functions. The gKCCA method has at least two model parameters: the Gram matrix regularization parameter $\varepsilon$ and other parameters belonging to the kernel function, such as Gaussian $\sigma$ or polynomial power $k$. To estimate these parameters, nonlinear cross validation was performed using the adjusted multiple coefficient of determination from an augmented linear model as the cross-validation metric. Experiments with different levels of noise, and different kernels, show that gKCCA is able to recover the underlying nonlinearity between two fields. The application of gKCCA to observed SST data (ERSST) shows a significant quadratic coupling between the low-pass (4-6 years) field and the high-pass (2-3 years) field for the leading gKCCA subspace. The high-frequency pattern has stronger anomalies in the central Pacific than in the east Pacific, compared to other studies investigating nonlinear interactions, but the methods used in these other studies were different. Future studies of these components using climate models are needed.

Further investigations of the application of gKCCA to nonlinear questions in climate science are needed. Further methodological experiments, including the application of a wider range of kernel functions (e.g., Laplacian, periodic, and Matern kernels), are also required. Finally, methodological developments are possible, such as the development of gKCCA for fields with nonlinear coupling between propagating components.

Acknowledgments. My Julia package CoupledFields contains source code for gKCCA (https:/github.com/ Mattriks/CoupledFields). Julia is a state-of-the-art programming language. We thank several anonymous reviewers for their useful suggestions that helped improve the manuscript.

\section{APPENDIX A}

\section{Factorizing the Polynomial and Gaussian Kernel}

First it is necessary to factorize the polynomial kernel $(\mathbf{u v})^{k}$, where here $\mathbf{u}$ and $\mathbf{v}$ are row vectors of $\mathbf{X}$ :

$$
\begin{aligned}
& \left(\mathbf{u v}^{\prime}\right)^{k} \\
& =\sum_{\text {All }}\left[\left(\frac{k !}{j_{1} ! \cdots j_{p} !}\right) u_{1}^{j_{1}} v_{1}^{j_{1}} \cdots u_{p}^{j_{p}} v_{p}^{j_{p}}\right] \\
& =\Phi_{k}(\mathbf{u}) \Phi_{k}(\mathbf{v})^{\prime} \\
& \therefore \Phi_{k}(\mathbf{x})=\left[\cdots, \sqrt{\left(\frac{k !}{j_{1} ! \cdots j_{p} !}\right)} x_{1}^{\left.j_{1} \cdots x_{p}^{j_{p}}, \ldots\right]},\right.
\end{aligned}
$$


where partition $\mathbf{j}=\left(j_{1}, \ldots, j_{p}\right)$ is a set of integers such that $j \geq 0$ and $\sum_{i=1}^{p} j_{i}=k$, and $\Phi_{k}(\cdot)$ is the feature space vector of the polynomial kernel. The length of $\Phi_{k}(\cdot)$ is $(k+p-1) ! /[k !(p-1) !]$

The Gaussian kernel $\exp \left(-\gamma\left\|\mathbf{x}_{i}-\mathbf{x}_{j}\right\|^{2}\right)$ is a function of two linear kernels and the sum of all polynomial kernels:

$$
\begin{aligned}
& \exp \left(-\gamma\left\|\mathbf{x}_{i}-\mathbf{x}_{j}\right\|^{2}\right) \\
& =\exp \left(-\gamma \mathbf{x}_{i} \mathbf{x}_{i}^{\prime}-\gamma \mathbf{x}_{j} \mathbf{x}_{j}^{\prime}\right) \exp \left(2 \gamma \mathbf{x}_{i} \mathbf{x}_{j}^{\prime}\right) \\
= & \exp \left(-\gamma \mathbf{x}_{i} \mathbf{x}_{i}^{\prime}-\gamma \mathbf{x}_{j} \mathbf{x}_{j}^{\prime}\right) \sum_{k=0}^{\infty}(2 \gamma)^{k}\left(\mathbf{x}_{i} \mathbf{x}_{j}^{\prime}\right)^{k} / k ! \\
& =\Phi\left(\mathbf{x}_{i}\right) \Phi\left(\mathbf{x}_{j}\right)^{\prime} \\
& \therefore \Phi(\mathbf{x})=e^{-\gamma \mathbf{x} \mathbf{x}^{\prime}}\left[\ldots, \sqrt{\frac{(2 \gamma)^{k}}{k !}} \Phi_{k}(\mathbf{x}), \ldots\right]
\end{aligned}
$$

because $e^{x}=\sum_{k=0}^{\infty} x^{k} / k$ !. Note that $\Phi(\cdot)$ (without the subscript $k$ ) is the feature space vector of the Gaussian kernel. The sum of polynomial kernels in Eq. (A2c) means that the Gaussian kernel feature space vector in Eq. (A2e) contains the monomials of all polynomial kernel feature space vectors. That is, the Gaussian kernel feature space vector is of infinite length.

All the above proves (for the polynomial and Gaussian kernels) that a Kernel matrix $\mathbf{G}=K(\cdot, \cdot)$ of a field $\mathbf{X}$, is the $n \times n$ Gram matrix of a feature space $\Phi(\mathbf{X})$. That is, $\mathbf{G}_{i j}=K\left(\mathbf{x}_{i}, \mathbf{x}_{j}\right)=\Phi\left(\mathbf{x}_{i}\right) \Phi\left(\mathbf{x}_{j}\right)^{\prime}$.

\section{APPENDIX B}

\section{Piecewise Linear Basis Matrix}

Piecewise linear basis matrix can be expressed as follows:

$$
\phi\left(\mathbf{R}_{j}\right)=\left(\begin{array}{cccc}
r_{1}-\mathrm{bp}_{1} & 0 & \ldots & 0 \\
\vdots & \vdots & \vdots & \vdots \\
r_{l}-\mathrm{bp}_{1} & 0 & \cdots & 0 \\
\mathrm{bp}_{2}-\mathrm{bp}_{1} & r_{l+1}-\mathrm{bp}_{2} & \cdots & 0 \\
\vdots & \vdots & \vdots & \vdots \\
\mathrm{bp}_{2}-\mathrm{bp}_{1} & r_{m}-\mathrm{bp}_{2} & \cdots & 0 \\
\mathrm{bp}_{2}-\mathrm{bp}_{1} & \mathrm{bp}_{3}-\mathrm{bp}_{2} & r_{m+1}-\mathrm{bp}_{3} & \vdots \\
\vdots & \vdots & \vdots & \vdots \\
\mathrm{bp}_{2}-\mathrm{bp}_{1} & \mathrm{bp}_{3}-\mathrm{bp}_{2} & \cdots & r_{n}-\mathrm{bp}_{\mathrm{df}}
\end{array}\right)
$$

where $\left(r_{1}, r_{2}, \ldots, r_{n}\right)$ are the elements of $\mathbf{R}_{j}$ (expressed here as if the elements were sorted into ascending order, for convenience-note for practical calculation sorting of rows is not required), $b p$ is a df-length vector of breakpoints, and df is the degrees of freedom. The rows of $\phi\left(\mathbf{R}_{j}\right)$ change with each breakpoint (i.e., $\mathrm{bp}_{1} \leq$ $r_{1: l}<\mathrm{bp}_{2}, \mathrm{bp}_{2}<r_{(l+1): m}<\mathrm{bp}_{3}$, and so forth). The degrees of freedom of the piecewise linear basis is controlled by the number of breakpoints; for example, for $\mathrm{df}=3$ the breakpoints are bp $=\left[\min \left(\mathbf{R}_{j}\right), q_{33}\left(\mathbf{R}_{j}\right), q_{66}\left(\mathbf{R}_{j}\right)\right]$, where $q_{k}(\cdot)$ refers to the $k \%$ quantile of $\mathbf{R}_{j}$. Note that if $\mathrm{df}=1$, then $\mathrm{bp}=\left[\min \left(\mathbf{R}_{j}\right)\right]$ and $\phi\left(\mathbf{R}_{j}\right)=\mathbf{R}_{j}$. Each column of $\phi\left(\mathbf{R}_{j}\right)$ represents a segment of a piecewise linear model.

\section{REFERENCES}

Arenas-García, J., K. B. Petersen, G. Camps-Valls, and L. K. Hansen, 2013: Kernel multivariate analysis framework for supervised subspace learning. IEEE Signal Process. Mag., 30, 16-29, doi:10.1109/MSP.2013.2250591.
Barnett, T. P., 1991: The interaction of multiple time scales in the tropical climate system. J. Climate, 4, 269-285, doi:10.1175/ 1520-0442(1991)004<0269:TIOMTS>2.0.CO;2.

Bejarano, L., and F.-F. Jin, 2008: Coexistence of equatorial coupled modes of ENSO. J. Climate, 21, 3051-3067, doi:10.1175/ 2007JCLI1679.1.

Bretherton, C., C. Smith, and J. M. Wallace, 1992: An intercomparison of methods for finding coupled patterns in climate. J. Climate, 5, 541-560, doi:10.1175/1520-0442(1992)005<0541: AIOMFF $>2.0 . \mathrm{CO} ; 2$.

Cannon, A. J., 2006: Nonlinear principal predictor analysis: Application to the Lorenz system. J. Climate, 19, 579-589, doi:10.1175/JCLI3634.1.

, 2009: Negative ridge regression parameters for improving the covariance structure of multivariate linear downscaling models. Int. J. Climatol., 29, 761-769, doi:10.1002/joc.1737.

, and W. W. Hsieh, 2008: Robust nonlinear canonical correlation analysis: application to seasonal climate forecasting. Nonlinear Processes Geophys., 15, 221-232, doi:10.5194/ npg-15-221-2008.

Chung, C., and S. Nigam, 1999: Weighting of geophysical data in principal component analysis. J. Geophys. Res., 104, 1692516 928, doi:10.1029/1999JD900234. 
DelSole, T., and M. K. Tippett, 2008: Predictable components and singular vectors. J. Atmos. Sci., 65, 1666-1678, doi:10.1175/ 2007JAS2401.1.

Evans, M. N., J. E. Smerdon, A. Kaplan, S. E. Tolwinski-Ward, and J. F. González-Rouco, 2014: Climate field reconstruction uncertainty arising from multivariate and nonlinear properties of predictors. Geophys. Res. Lett., 41, 9127-9134, doi:10.1002/ 2014 GL062063.

Fukumizu, K., and C. Leng, 2014: Gradient-based kernel dimension reduction for regression. J. Amer. Stat. Assoc., 109, 359-370, doi:10.1080/01621459.2013.838167.

Hsieh, W. W., 2001: Nonlinear canonical correlation analysis of the tropical Pacific climate variability using a neural network approach. J. Climate, 14, 2528-2539, doi:10.1175/ 1520-0442(2001)014<2528:NCCAOT>2.0.CO;2.

Huang, B., and Coauthors, 2016: Further exploring and quantifying uncertainties for Extended Reconstructed Sea Surface Temperature (ERSST) version 4 (v4). J. Climate, 29, 3119-3142, doi:10.1175/JCLI-D-15-0430.1.

Lim, Y., S. Jo, J. Lee, H.-S. Oh, and H.-S. Kang, 2012: An improvement of seasonal climate prediction by regularized canonical correlation analysis. Int. J. Climatol., 32, 1503-1512, doi:10.1002/joc. 2368 .

Lima, C. H. R., U. Lall, T. Jebara, and A. G. Barnston, 2009: Statistical prediction of ENSO from subsurface sea temperature using a nonlinear dimensionality reduction. J. Climate, 22, 4501-4519, doi:10.1175/2009JCLI2524.1.

Martínez-Gómez, E., M. T. Richards, and D. S. P. Richards, 2014: Distance correlation measures for discovering associations in large astrophysical databases. Astrophys. J., 781, 39, doi:10.1088/0004-637X/781/1/39.

Monahan, A. H., and A. Dai, 2004: The spatial and temporal structure of ENSO nonlinearity. J. Climate, 17, 3026-3036, doi:10.1175/1520-0442(2004)017<3026:TSATSO>2.0.CO;2.

NOAA, 2017: Extended Reconstructed Sea Surface Temperature, version 4. NOAA/OAR/ESRL/PSD, accessed 16 January 2017. [Available online at http://www.esrl.noaa.gov/psd/data/ gridded.]

Ortiz Beviá, M. J., I. Pérez-González, F. J. Alvarez-García, and A. Gershunov, 2010: Nonlinear estimation of El Niño impact on the North Atlantic winter. J. Geophys. Res., 115, D21123, doi:10.1029/2009JD013387.
Ramírez, M. C., N. J. Ferreira, and H. F. C. Velho, 2006: Linear and nonlinear statistical downscaling for rainfall forecasting over southeastern Brazil. Wea. Forecasting, 21, 969-989, doi:10.1175/WAF981.1.

Roulston, M. S., and J. D. Neelin, 2003: Nonlinear coupling between modes in a low-dimensional model of ENSO. Atmos.Ocean, 41, 217-231, doi:10.3137/ao.410303.

Schreiber, T., and A. Schmitz, 2000: Surrogate time series. Physica $D$, 142, 346-382, doi:10.1016/S0167-2789(00)00043-9.

Schreier, P. J., 2008: A unifying discussion of correlation analysis for complex random vectors. IEEE Trans. Signal Process., 56 , 1327-1336, doi:10.1109/TSP.2007.909054.

Scott, D. W., 2015: The curse of dimensionality and dimension reduction. Multivariate Density Estimation: Theory, Practice, and Visualization, D. W. Scott, Ed., John Wiley and Sons, 217 240, doi:10.1002/9781118575574.ch7.

Smerdon, J. E., A. Kaplan, D. Chang, and M. N. Evans, 2011: A pseudoproxy evaluation of the CCA and RegEM methods for reconstructing climate fields of the last millennium. J. Climate, 24, 1284-1309, doi:10.1175/2010JCLI4110.1.

Smoliak, B. V., J. M. Wallace, P. Lin, and Q. Fu, 2015: Dynamical adjustment of the Northern Hemisphere surface air temperature field: Methodology and application to observations. J. Climate, 28, 1613-1629, doi:10.1175/JCLI-D-14-00111.1.

Tippett, M. K., T. DelSole, S. J. Mason, and A. G. Barnston, 2008: Regression-based methods for finding coupled patterns. J. Climate, 21, 4384-4398, doi:10.1175/2008JCLI2150.1.

Tolwinski-Ward, S. E., M. N. Evans, M. K. Hughes, and K. J. Anchukaitis, 2011: An efficient forward model of the climate controls on interannual variation in tree-ring width. Climate Dyn., 36, 2419-2439, doi:10.1007/s00382-010-0945-5.

Wilks, D. S., 2008: Improved statistical seasonal forecasts using extended training data. Int. J. Climatol., 28, 1589-1598, doi:10.1002/joc.1661.

Wu, A., W. W. Hsieh, and A. Shabbar, 2005: The nonlinear patterns of North American winter temperature and precipitation associated with ENSO. J. Climate, 18, 1736-1752, doi:10.1175/ JCLI3372.1.

Yang, X.-Q., Q. Xie, and S.-S. Huang, 1997: Unstable interannual oscillation modes in a linearized coupled ocean-atmosphere model and their association with ENSO. Meteor. Atmos. Phys., 62, 161-177, doi:10.1007/BF01029700. 